IJMMS 32:10 (2002) 587-614

PII. S0161171202202069

http://ijmms.hindawi.com

(c) Hindawi Publishing Corp.

\title{
ON THE COMPATIBLE WEAKLY NONLOCAL POISSON BRACKETS OF HYDRODYNAMIC TYPE
}

\author{
ANDREI YA. MALTSEV
}

Received 5 February 2002

\begin{abstract}
We consider the pairs of general weakly nonlocal Poisson brackets of hydrodynamic type (Ferapontov brackets) and the corresponding integrable hierarchies. We show that, under the requirement of the nondegeneracy of the corresponding "first" pseudo-Riemannian metric $g_{(0)}^{v \mu}$ and also some nondegeneracy requirement for the nonlocal part, it is possible to introduce a "canonical" set of "integrable hierarchies" based on the Casimirs, momentum functional and some "canonical Hamiltonian functions." We prove also that all the "higher" "positive" Hamiltonian operators and the "negative" symplectic forms have the weakly nonlocal form in this case. The same result is also true for "negative" Hamiltonian operators and "positive" symplectic structures in the case when both pseudo-Riemannian metrics $g_{(0)}^{v \mu}$ and $g_{(1)}^{v \mu}$ are nondegenerate.
\end{abstract}

2000 Mathematics Subject Classification: 35Q58, 37K05, 37K10, 37K25.

1. Introduction. We discuss in this paper the Poisson pencils of weakly nonlocal Poisson brackets of hydrodynamic type (Ferapontov brackets). This means that we consider the following pair of Hamiltonian operators:

$$
\begin{aligned}
& \hat{J}_{(0)}^{v \mu}=g_{(0)}^{v \mu}(U) \frac{d}{d X}+b_{(0) \eta}^{\nu \mu}(U) U_{X}^{\eta}+\sum_{k=1}^{g_{0}} e_{(0) k} w_{(0) k \eta}^{v}(U) U_{X}^{\eta} D^{-1} w_{(0) k \zeta}^{\mu}(U) U_{X}^{\zeta}, \\
& \hat{J}_{(1)}^{\nu \mu}=g_{(1)}^{\nu \mu}(U) \frac{d}{d X}+b_{(1) \eta}^{v \mu}(U) U_{X}^{\eta}+\sum_{k=1}^{g_{1}} e_{(1) k} w_{(1) k \eta}^{v}(U) U_{X}^{\eta} D^{-1} w_{(1) k \zeta}^{\mu}(U) U_{X}^{\zeta},
\end{aligned}
$$

where $e_{(0) k}, e_{(1) k}= \pm 1$ and $D^{-1}=(d / d X)^{-1}$ are defined in a "skew-symmetric" way

$$
D^{-1}=\frac{1}{2}\left[\int_{-\infty}^{X} d X-\int_{X}^{+\infty} d X\right]
$$

and require that the expression

$$
\hat{J}_{\lambda}^{v \mu}=\hat{J}_{(0)}^{v \mu}+\lambda \hat{J}_{(1)}^{v \mu}
$$

defines the Poisson bracket satisfying Jacobi identity for any $\lambda$.

We mention here that the brackets of this kind are the generalization of DubrovinNovikov local homogeneous brackets of hydrodynamic type $[6,7,8]$ :

$$
\left\{U^{v}(X), U^{\mu}(Y)\right\}=g^{\nu \mu}(U) \delta^{\prime}(X-Y)+b_{\eta}^{\nu \mu}(U) U_{X}^{\eta} \delta(X-Y)
$$


with the Hamiltonian operator

$$
\hat{J}_{D N}^{v \mu}=g^{v \mu}(U) \frac{d}{d X}+b_{\eta}^{v \mu}(U) U_{X}^{\eta}
$$

Theorem 1.1 (Dubrovin and Novikov). Consider the bracket (1.4) with nondegenerate tensor $g^{v \mu}(U)$. From the Leibnitz identity, it follows that $g^{v \mu}(U)$ and $\Gamma_{v \eta}^{\mu}(U)=-g_{v \xi}(U) b_{\eta}^{\xi \mu}(U)\left(g_{v \xi} g^{\xi \mu}=\delta_{v}^{\mu}\right)$ transforms as a metric with upper indices and the Christoffel symbols under the pointwise coordinate transformations $\tilde{U}^{v}=\tilde{U}^{v}(U)$.

Bracket (1.4) is skew-symmetric if and only if $\mathrm{g}^{\nu \mu}$ is symmetric and the connection $\Gamma_{v \eta}^{\mu}$ is compatible with the metric $\nabla_{\eta} g^{v \mu} \equiv 0$.

Bracket (1.4) satisfies the Jacobi identity if and only if the connection $\Gamma_{v \eta}^{\mu}$ is symmetric and has zero curvature $R_{\eta \xi}^{v \mu} \equiv 0$.

It follows from Dubrovin-Novikov theorem that any bracket (1.4) with nondegenerate $g^{\nu \mu}$ can be written locally in the "constant form"

$$
\left\{n^{\nu}(X), n^{\mu}(Y)\right\}=\epsilon^{v} \delta^{v \mu} \delta^{\prime}(X-Y), \quad \epsilon^{v}= \pm 1
$$

in the flat coordinates $n^{v}=n^{v}(U)$.

The functionals

$$
N^{v}=\int_{-\infty}^{+\infty} n^{v}(X) d X
$$

are Casimirs of bracket (1.4) and the functional

$$
P=\int_{-\infty}^{+\infty} \frac{1}{2} \sum_{v=1}^{N} \epsilon^{v} n^{v}(X) n^{v}(X) d X
$$

is a momentum operator generating the flow $U_{T}^{v}=U_{X}^{v}$. Form (1.6) can be considered as the canonical form for the DN-bracket (1.4) with the nondegenerate tensor $g^{v \mu}$.

It can be also seen that any functional of "hydrodynamic type"

$$
H=\int_{-\infty}^{+\infty} h(U) d X
$$

generates a "hydrodynamic type system"

$$
U_{T}^{v}=V_{\mu}^{v}(U) U_{X}^{\mu}
$$

according to bracket (1.4).

We also mention that bracket (1.4) with degenerate tensor $g^{\nu \mu}(U)$ of constant rank has more complicated but also nice differential geometric structure (see [18]).

The first generalization of DN-bracket to the weakly nonlocal case was the MokhovFerapontov bracket [29]

$$
\left\{U^{v}(X), U^{\mu}(Y)\right\}=g^{\nu \mu}(U) \delta^{\prime}(X-Y)+b_{\eta}^{v \mu}(U) U_{X}^{\eta} \delta(X-Y)+c U_{X}^{v} v(X-Y) U_{Y}^{\mu},
$$


where $v(X-Y)=1 / 2 \operatorname{sgn}(X-Y)$, corresponding to the Hamiltonian operator

$$
\hat{J}_{D N}^{v \mu}=g^{v \mu}(U) \frac{d}{d X}+b_{\eta}^{v \mu}(U) U_{X}^{\eta}+c U_{X}^{v} D^{-1} U_{X}^{\mu}
$$

THEOREM 1.2 (Mokhov and Ferapontov). Consider bracket (1.11) with nondegenerate tensor $g^{v \mu}(U)$. Then

(1) bracket (1.11) is skew-symmetric and satisfies Leibnitz identity if and only if the tensor $g^{\nu \mu}(U)$ is a metric with upper indices and $\Gamma_{v \eta}^{\mu}=-g_{\nu \xi} b_{\eta}^{\xi \mu}$ are the connection coefficients compatible with $g^{v \mu}(U)$;

(2) bracket (1.11) satisfies the Jacobi identity if and only if the connection $\Gamma_{v \eta}^{\mu}$ is symmetric and has the constant curvature equal to $c$, that is,

$$
R_{\mu \eta}^{v \tau}=c\left(\delta_{\mu}^{v} \delta_{\eta}^{\tau}-\delta_{\mu}^{\tau} \delta_{\eta}^{v}\right)
$$

Bracket (1.11) has a weakly nonlocal form. However, any local translationally invariant functional

$$
H=\int h(U) d X
$$

generates a local system of hydrodynamic type with respect to (1.11). Indeed, we have

$$
U_{X}^{\mu} \frac{\partial h}{\partial U^{\mu}} \equiv \partial_{X} h
$$

if $h$ does not depend on $X$ explicitly; so, the application of $D^{-1}$ gives the local expression for the corresponding flow.

The canonical form of bracket (1.11) was first presented by Pavlov in [31] and can be written as

$$
\begin{aligned}
\left\{n^{\nu}(X), n^{\mu}(Y)\right\}= & \left(\epsilon^{v} \delta^{\nu \mu}-c n^{\nu} n^{\mu}\right) \delta^{\prime}(X-Y)-c n_{X}^{v} n^{\mu} \delta(X-Y) \\
& +c n_{X}^{v} v(X-Y) n_{Y}^{\mu},
\end{aligned}
$$

where $n^{v}=n^{v}(U)$ are the annihilators for bracket (1.11) (on the space of rapidly decreasing functions $n^{v}(X)$ at $\left.X \rightarrow \pm \infty\right)$. Also, the implicit expression for the density of $P$ was represented in [31].

We will see, however, that the Casimirs and the momentum operator for bracket (1.11) actually depend on the boundary conditions imposed on the functions $U^{v}(X)$ for $X \rightarrow \pm \infty$ (see [25]) (the condition $U^{v} \rightarrow 0, X \rightarrow \pm \infty$, in general, is not invariant under the pointwise transformations $\left.\tilde{U}^{v}=\tilde{U}^{v}(U)\right)$. As pointed out in [25], we cannot speak about the Casimirs and momentum functional until we fix the boundary conditions at infinity, and, in the general case, it is better to speak about the invariant set of $N+1$ (for MF-bracket) functionals playing the role of either Casimirs of momentum operator according to the boundary conditions. We consider this later for the case of more general Ferapontov brackets. 
The general Ferapontov bracket $[11,12,13,16]$ has the form

$$
\begin{aligned}
\left\{U^{\nu}(X), U^{\mu}(Y)\right\}= & g^{v \mu}(U) \delta^{\prime}(X-Y)+b_{\eta}^{\nu \mu}(U) U_{X}^{\eta} \delta(X-Y) \\
& +\sum_{k=1}^{g} e_{k} w_{k \eta}^{v}(U) U_{X}^{\eta} v(X-Y) w_{k \zeta}^{\mu}(U) U_{Y}^{\zeta},
\end{aligned}
$$

$e_{k}= \pm 1$, which corresponds to the weakly nonlocal Hamiltonian operator

$$
\hat{J}_{F}^{v \mu}=g^{v \mu}(U) \frac{d}{d X}+b_{\eta}^{v \mu}(U) U_{X}^{\eta}+\sum_{k=1}^{g} e_{k} w_{k \eta}^{v}(U) U_{X}^{\eta} D^{-1} w_{k \zeta}^{\mu}(U) U_{X}^{\zeta} .
$$

THEOREM 1.3 (Ferapontov [11, 16]). Consider bracket (1.17) with nondegenerate tensor $g^{\nu \mu}(U)$. Then,

(1) bracket (1.17) is skew-symmetric and satisfies Leibnitz identity if and only if tensor $g^{\nu \mu}(U)$ is a metric with upper indices and $\Gamma_{v \eta}^{\mu}=-g_{\nu \xi} b_{\eta}^{\xi \mu}$ are the connection coefficients compatible with $g^{\nu \mu}(U)$;

(2) bracket (1.11) satisfies the Jacobi identity if and only if the connection $\Gamma_{v \eta}^{\mu}$ is symmetric and the metric $g^{v \mu}$ and tensors $w_{k \eta}^{v}(U)$ satisfy the equations

$$
\begin{aligned}
g^{\nu \tau} w_{k \tau}^{\mu} & =g^{\mu \tau} w_{k \tau}^{v}, \quad \nabla_{\nu} w_{k \eta}^{\mu}=\nabla_{\eta} w_{k \nu}^{\mu}, \\
R_{\mu \eta}^{v \tau} & =\sum_{k=1}^{g} e_{k}\left(w_{k \mu}^{v} w_{k \eta}^{\tau}-w_{k \mu}^{\tau} w_{k \eta}^{v}\right) .
\end{aligned}
$$

Moreover, this set is commutative $\left[w_{k}, w_{k^{\prime}}\right]=0$.

It was pointed out by Ferapontov that the equations written above are Gauss and Petersson-Codazzi equations for the submanifold $\mu^{N}$ with flat normal connection in the pseudo-Euclidean space $E^{N+g}$. In this consideration, the tensor $g^{v \mu}$ is the first quadratic form of $\mathcal{M}^{N}$, and $w_{k \eta}^{v}$ are the Weingarten operators corresponding to $g$ parallel vector fields in the normal bundle $\mathbf{N}_{k}$, such that $\left\langle\mathbf{N}_{k}, \mathbf{N}_{m}\right\rangle=e_{k} \delta_{k m}$. It was also proved by Ferapontov that these brackets can be constructed as a Dirac restriction of the local DN-bracket

$$
\left\{Z^{I}(X), Z^{J}(Y)\right\}=\epsilon^{I} \delta^{I J} \delta^{\prime}(X-Y), \quad I, J=1, \ldots, N+g, \epsilon^{I}= \pm 1
$$

in $E^{N+g}$ to the submanifold $\mathcal{M}^{N}[12,16]$.

As far as we know, the cases of brackets (1.11), (1.17) with the degenerate tensors $g^{v \mu}(U)$ were not studied in the literature.

All brackets (1.4), (1.11), and (1.17) are closely connected with the diagonalizable integrable systems (1.10).

The general procedure of integration of the so-called "semi-Hamiltonian" diagonal systems of hydrodynamic type was constructed by Tsarëv [34, 35]. It can be shown that any diagonal system (1.10) which is Hamiltonian with respect to bracket (1.4), (1.11), or (1.17) (with diagonal $g^{v \mu}(U)$ ) satisfies Tsarëv "semi-Hamiltonian" property, and so, it can be integrated by Tsarëv's method. Probably, all semi-Hamiltonian systems are in fact Hamiltonian corresponding to some weakly nonlocal H.T.P.B. with (maybe) an infinite number of terms in the nonlocal tail. Some investigation of this problem 
can be found in [3, 16], but, in general, this problem is still open. We also mention that the examples of nondiagonalizable Hamiltonian integrable (by inverse scattering methods) systems (1.10) were also investigated in [14, 15].

As was pointed out in $[13,16]$, if the manifold $M^{N}$ has a holonomic net of lines of curvature, the metric $g^{v \mu}(U)$ and all the operators $w_{k \eta}^{v}$ can be written in the diagonal form in the corresponding coordinates $r^{v}=r^{v}(U)$. Here, we do not impose this requirement and consider any brackets of Ferapontov type.

We will assume that the flows $w_{k \eta}^{v}(U) U_{X}^{\eta}$ in the nonlocal part of (1.17) are linearly independent (with constant coefficients). (The nonlocal part in (1.17) actually represents the nondegenerate quadratic form on the linear space generated by $w_{k \eta}^{v}(U) U_{X}^{\eta}$, $k=1, \ldots, g$ written in the canonical form with $e_{k}= \pm 1$.) As pointed out by Ferapontov, the local functional

$$
H=\int h(U) d X
$$

generates in this case the local flow with respect to bracket (1.17) if and only if the functional $H$ is a conservation law for any of the flows $w_{k \eta}^{v}(U) U_{X}^{\eta}$ such that the expressions

$$
w_{k \eta}^{v}(U) U_{X}^{\eta} \frac{\partial h}{\partial U^{v}}
$$

represent the total derivatives with respect to $X$ of some functions $Q_{k}(U)$ for any $k$.

This fact is also true for more general weakly nonlocal Poisson brackets having the form

$$
\begin{aligned}
\left\{\varphi^{i}(x), \varphi_{j}(y)\right\}= & \sum_{k=1}^{G} B_{k}^{i j}\left(\varphi, \varphi_{x}, \ldots\right) \delta^{(k)}(x-y) \\
& +\sum_{k=1}^{g} e_{k} S_{k}^{i}\left(\varphi, \varphi_{x}, \ldots\right) \nu(x-y) S_{k}^{j}\left(\varphi, \varphi_{y}, \ldots\right),
\end{aligned}
$$

where $\delta^{(k)}(x-y)=(d / d x)^{k} \delta(x-y), e_{k}= \pm 1$, and the set $\left\{S_{k}^{i}\left(\varphi, \varphi_{x}, \ldots\right)\right\}$ is linearly independent.

As far as we know, the first example written precisely in this form was the Sokolov bracket [33]

$$
\{\varphi(x), \varphi(y)\}=-\varphi_{x} v(x-y) \varphi_{y}
$$

for the Krichever-Novikov equation

$$
\varphi_{t}=\varphi_{x x x}-\frac{3}{2} \frac{\varphi_{x x}^{2}}{\varphi_{x}}+\frac{h(\varphi)}{\varphi_{x}}
$$


where $h(\varphi)=c_{3} \varphi^{3}+c_{2} \varphi^{2}+c_{1} \varphi+c_{0}$, with the Hamiltonian function

$$
H=\int\left(\frac{1}{2} \frac{\varphi_{x x}^{2}}{\varphi_{x}^{2}}+\frac{1}{3} \frac{h(\varphi)}{\varphi_{x}^{2}}\right) d x .
$$

As established in [23, 24], the flows $S_{k}^{i}\left(\varphi, \varphi_{x}, \ldots\right)$ commute with each other for any general bracket (1.23) and conserve the corresponding Hamiltonian structure (1.23) on the phase space $\left\{\varphi^{i}(x)\right\}$ (this fact was important for the averaging procedure for such brackets considered there). However, for general brackets (1.23), they are not necessarily generated by the local Hamiltonian functions having the form

$$
H=\int h\left(\varphi, \varphi_{x}, \ldots\right) d x
$$

Actually, brackets (1.23) are very common for so-called "integrable systems" (like $\mathrm{KdV}$ or NLS) possessing the multi-Hamiltonian structures connected by the recursion operator according to the Lenard-Magri scheme [20]. As such, it was proved in [10] that all the higher PB brackets for KdV given by the recursion scheme starting from Gardner-Zakharov-Faddev bracket

$$
\{\varphi(x), \varphi(y)\}=\delta^{\prime}(x-y)
$$

and the Magri bracket

$$
\{\varphi(x), \varphi(y)\}=-\delta^{\prime \prime \prime}(x-y)+4 \varphi(x) \delta^{\prime}(x-y)+2 \varphi_{x} \delta(x-y)
$$

have exactly form (1.23). In [25], the same fact was proved for the case of NLS hierarchy, and also the weakly nonlocal form of the "negative" symplectic forms for KdV and NLS was established.

Brackets (1.4), (1.11), and (1.17) appear in these systems as the "dispersionless" limit of the corresponding bracket (1.23) or, in more general case, as a result of the averaging of (1.23) on the families of quasiperiodic solutions of corresponding evolution system connected with the Whitham method for slow modulations of parameters $[1,2,6,7,8,21,22,23,24,26,30,32]$.

We consider here the compatible brackets of Ferapontov type and prove the similar facts for the case of the nondegenerate pencils (i.e., $\operatorname{det} g_{(0)}^{\nu \mu} \neq 0$ ) with also some nondegeneracy conditions for nonlocal part of $\hat{J}_{(0)}+\lambda \hat{J}_{(1)}$.

We also mention that the wide classes of local pencils of hydrodynamic type (DNbrackets) were investigated in detail in [4] (see also [5, 9] and the references therein), where they play an important role in the structure of Dubrovin-Frobenius manifolds connected with solutions of WDVV equation for topological field theories. In [16, 27], some important questions of weakly nonlocal pencils of hydrodynamic type (H.T.) were also considered. In [17, 28], also the generic diagonal compatible flat pencils in terms of inverse scattering method (see $[19,36]$ ) were discussed.

2. On the canonical form and symplectic operator for the general $F$-bracket. We now formulate the properties of brackets (1.17) established in [25] which we will need in further consideration. 
Consider bracket (1.17) with nondegenerate tensor $g^{v \mu}(U)$. According to Ferapontov results, we can represent it as a Dirac restriction of DN-bracket in $\mathbb{R}^{N+g}$ to the submanifold $\mu^{N} \subset \mathbb{R}^{N+g}$ with flat normal connection. We fix the point $z \in \mathcal{M}^{N}$ and introduce the corresponding loop space in the vicinity of $z$

$$
L\left(\mathcal{M}^{N}, z\right)=\left\{\gamma: \mathbb{R}^{1} \rightarrow \mathcal{M}^{N}: \gamma(-\infty)=\gamma(+\infty)=z \in \mathcal{M}^{N}\right\}
$$

So, we fix the boundary conditions for the functions $U^{v}(X)$ and require that these functions rapidly approach their boundary values at $X \rightarrow \pm \infty$.

Theorem 2.1 (Maltsev and Novikov [25]). Consider the bracket (1.17) with nondegenerate $g^{v \mu}(U)$ defined on the loop space $L\left(\mu^{N}, z\right)$. Consider the corresponding embedding $\mu^{N} \subset \mathbb{R}^{N+g}$ with flat normal connection. Consider the flat orthogonal coordinates $Z^{I}$ in $\mathbb{R}^{N+g}$ such that

(1) $Z^{I}(z)=0, I=1, \ldots, N+g$, and the corresponding DN-bracket in $\mathbb{R}^{N+g}$ has the form

$$
\left\{Z^{I}(X), Z^{J}(Y)\right\}=E^{I} \delta^{I J} \delta^{\prime}(X-Y), \quad I, J=1, \ldots, N+g, E^{I}= \pm 1
$$

(2) the first $N$ coordinates $Z^{1}, \ldots, Z^{N}$ are tangential to $\mathcal{M}^{N}$ at the point $z$;

(3) the last $g$ coordinates $Z^{N+1}, \ldots, Z^{N+g}$ are orthogonal to $M^{N}$ at the point $z$.

Then,

(1) bracket (1.17) has exactly $N$ local Casimirs given by the functionals

$$
N^{v}=\int_{-\infty}^{+\infty} n^{v}(X) d X
$$

where $n^{v}(U)$ are the restrictions of the first $N$ (tangential to $\mu^{N}$ at $z$ ) coordinates $Z^{1}, \ldots, Z^{N}$ on $\mu^{N}$,

(2) all the flows

$$
U_{t_{k}}^{v}=w_{k \eta}^{v}(U) U_{X}^{\eta}
$$

are Hamiltonian with respect to (1.17) with local Hamiltonian functionals

$$
H_{k}=\int_{-\infty}^{+\infty} h_{k}(X) d X, \quad k=1, \ldots, g,
$$

where $h_{k}(U)$ are the restriction of the last $g$ coordinates $Z^{N+k}, k=1, \ldots, g$ on $\mu^{N}$ and $w_{k \eta}^{v}(U)$ are the Weingarten operators corresponding to parallel vector fields $\mathbf{N}_{k}(U)$ in the normal bundle with the normalization

$$
\mathbf{N}_{k}(z)=\left(0, \ldots, 0,-E^{N+k}, 0, \ldots, 0\right)^{T},
$$

where $-E^{N+k}$ stays at the position $N+k$, in the coordinates $Z^{1}, \ldots, Z^{N+g}$, so $\left\langle\mathbf{N}_{k}(U), \mathbf{N}_{l}(U)\right\rangle=E^{N+k} \delta_{k l}$ (there is an arithmetic mistake in the journal variant of [25] where the generated flows are written as $\left.E^{N+k} w_{k \eta}^{v}(U) U_{X}^{\eta}\right)$, 
(3) bracket (1.17) has in coordinates $n^{v}(U)$ the "canonical form" corresponding to the space $L\left(\mu^{N}, z\right)$, that is,

$$
\begin{aligned}
\left\{n^{v}(X), n^{\mu}(Y)\right\}= & \left(\epsilon^{v} \delta^{v \mu}-\sum_{k=1}^{g} e_{k} f_{k}^{v}(n) f_{k}^{\mu}(n)\right) \delta^{\prime}(X-Y) \\
& -\sum_{k=1}^{g} e_{k}\left(f_{k}^{v}(n)\right)_{X} f_{k}^{\mu}(n) \delta(X-Y) \\
& +\sum_{k=1}^{g} e_{k}\left(f_{k}^{v}(n)\right)_{X} v(X-Y)\left(f_{k}^{\mu}(n)\right)_{Y},
\end{aligned}
$$

where $\epsilon^{v}=E^{v}, v=1, \ldots, N, e_{k}=E^{N+k}, k=1, \ldots, g, n^{v}(z)=0, f_{k}^{v}(z)=0$ (i.e., $f_{k}^{v}(0)=0$ ). (Actually, we have the equality $f_{k}^{v}(U) \equiv N_{k}^{v}(U)$, where $N_{k}^{v}(U)$ are the first $N$ components of the vectors $\mathbf{N}_{k}(U)$ in the coordinates $Z^{1}, \ldots, Z^{N+g}$.)

We note here that the Casimirs and "canonical functionals," as well as the canonical forms, depend on the phase space $L\left(\mu^{N}, z\right)$. So, if we do not fix the loop space, it is better to speak just about the $N+g$ canonical functions (the restrictions of the flat coordinates from $\mathbb{R}^{N+g}$ ) playing the role of Casimirs or canonical functionals depending on the boundary conditions. (Also, we will have many "canonical forms" of bracket (1.17) with different $f_{k}^{v}(U)$ in this case.)

For the case of Mokhov-Ferapontov bracket, the canonical form will be (1.16) for any fixed space $L\left(\mathcal{M}^{N}, z\right)$ (although the coordinates $n^{v}(U)$ will be different for different loop spaces). The explicit form of canonical functional, which is the momentum operator in this case, can then be written as (see [25])

$$
P=\frac{1}{c} \int_{-\infty}^{+\infty}\left(1-\sqrt{1-c \sum_{v=1}^{N} \epsilon^{v} n^{v} n^{v}}\right) d X .
$$

Using the restriction of the symplectic form of DN-bracket on $\mu^{N}$, it is also possible to get the symplectic form $\Omega_{v \mu}(X, Y)$ for the $F$-bracket (1.17) with nondegenerate $g^{v \mu}(U)$ [25]. The symplectic form appears to be also weakly nonlocal and can be written as

$$
\begin{aligned}
\Omega_{v \mu}(X, Y) & =\sum_{I=1}^{N+g} E^{I} \frac{\partial Z^{I}(U)}{\partial U^{v}}(X) v(X-Y) \frac{\partial Z^{I}(U)}{\partial U^{\mu}}(Y) \\
& =\sum_{\tau=1}^{N} \epsilon^{\tau} \frac{\partial n^{\tau}}{\partial U^{v}}(X) v(X-Y) \frac{\partial n^{\tau}}{\partial U^{\mu}}(Y)+\sum_{k=1}^{g} e_{k} \frac{\partial h_{k}}{\partial U^{v}}(X) v(X-Y) \frac{\partial h_{k}}{\partial U^{\mu}}(Y)
\end{aligned}
$$

on $\mu^{N}$.

We can also write the corresponding symplectic operator $\hat{\Omega}_{v \mu}$ on $L\left(\mathcal{M}^{N}, z\right)$ as

$$
\hat{\Omega}_{v \mu}=\sum_{\tau=1}^{N} \epsilon^{\tau} \frac{\partial n^{\tau}}{\partial U^{v}} D^{-1} \frac{\partial n^{\tau}}{\partial U^{\mu}}+\sum_{k=1}^{g} e_{k} \frac{\partial h_{k}}{\partial U^{v}} D^{-1} \frac{\partial h_{k}}{\partial U^{\mu}}
$$

with $D^{-1}$ defined in the skew-symmetric way. 
We introduce the space $\mathscr{V}_{0}(z)$ of vector fields $\xi^{v}(X)$ rapidly decreasing at $X \rightarrow \pm \infty$ on $L\left(M^{N}, z\right)$. It is easy to see that all the hydrodynamic type systems (1.10) satisfy this requirement as the vector fields on $L\left(\mu^{N}, z\right)$ and so belong to $\mathscr{V}_{0}(z)$. We can then naturally define the action of symplectic operator $\hat{\Omega}_{v \mu}$ on the space $\mathscr{V}_{0}(z)$.

We will also need the space $\mathscr{F}_{0}(z)$ of 1 -forms $\omega_{v}(X)$ rapidly decreasing at $X \rightarrow \pm \infty$ on $L\left(\mathcal{M}^{N}, z\right)$. We note here that $\hat{\Omega}_{v \mu} \xi^{\mu}(X) \notin \mathscr{F}_{0}(z)$ in the general case.

We will now prove by the direct calculation that the symplectic form $\hat{\Omega}_{v \mu}$ is the inverse of the Hamiltonian operator $\hat{J}_{F}^{v \mu}$ on the appropriate functional spaces.

THEOREM 2.2. (I) On the functional space $\mathscr{V}_{0}(z)$, the relation

$$
\hat{J}_{F}^{\nu \xi} \hat{\Omega}_{\xi \mu}=\hat{I}
$$

is true, where $\hat{I}$ is the identity operator on the space $\mathscr{V}_{0}(z)$.

(II) On the functional space $\mathscr{F}_{0}(z)$, the relation

$$
\hat{\Omega}_{v \xi} \hat{J}_{F}^{\xi \mu}=\hat{I}
$$

is true, where $\hat{I}$ is the identity operator on $\mathscr{F}_{0}(z)$.

Proof. (I) We have

$$
\begin{aligned}
\hat{J}_{F}^{\nu \xi} \hat{\Omega}_{\xi \mu}= & {\left[g^{\nu \xi}(U) \frac{d}{d X}+b_{\eta}^{\nu \xi}(U) U_{X}^{\eta}+\sum_{k=1}^{g} e_{k} w_{k \eta}^{v}(U) U_{X}^{\eta} D^{-1} w_{k \zeta}^{\xi}(U) U_{X}^{\zeta}\right] } \\
& \times\left[\sum_{\tau=1}^{N} \epsilon^{\tau} \frac{\partial n^{\tau}}{\partial U^{\xi}} D^{-1} \frac{\partial n^{\tau}}{\partial U^{\mu}}+\sum_{l=1}^{g} e_{l} \frac{\partial h^{l}}{\partial U^{\xi}} D^{-1} \frac{\partial h^{l}}{\partial U^{\mu}}\right],
\end{aligned}
$$

where the operators $w_{k \eta}^{v}(U)$ correspond to the vector fields $\mathbf{N}_{(k)}(U)$ such that

$$
\mathbf{N}_{(k)}(z)=\left(0, \ldots, 0,-e_{k}, 0, \ldots, 0\right)^{T}
$$

$\left(-e_{k}\right.$ stays at the position $\left.N+k\right)$, and we have identically $\left\langle\mathbf{N}_{(k)}(U), \mathbf{N}_{(l)}(U)\right\rangle=e_{k} \delta_{k l}$.

Since the integrals of $n^{\tau}(U)$, and $h^{l}(U)$ generate the local flows according to $\hat{J}_{F}^{v \mu}$, we have

$$
w_{k \zeta}^{\xi}(U) U_{X}^{\zeta} \frac{\partial n^{\tau}}{\partial U^{\xi}} \equiv\left(q_{k}^{\tau}(U)\right)_{X}, \quad w_{k \zeta}^{\xi}(U) U_{X}^{\zeta} \frac{\partial h^{l}}{\partial U^{\xi}} \equiv\left(p_{k}^{l}(U)\right)_{X}
$$

for some functions $q_{k}^{\tau}(U)$ and $p_{k}^{l}(U)$. Moreover, consider $N$ tangential vectors to $\mu_{N}$ in $\mathbb{R}^{N+g}$ corresponding to the coordinates $U^{v}$

$$
\mathbf{e}_{(\xi)}(U)=\left(\frac{\partial n^{1}}{\partial U^{\xi}}, \ldots, \frac{\partial n^{N}}{\partial U^{\xi}}, \frac{\partial h^{1}}{\partial U^{\xi}}, \ldots, \frac{\partial h^{g}}{\partial U^{\xi}}\right)^{T}
$$

and our parallel orthogonal vector fields $\mathbf{N}_{(k)}(U)$ defined by condition (2.14) in the coordinates $\left(Z^{1}, \ldots, Z^{N+g}\right)$. 
Since by the following definition:

$$
\frac{d}{d X} \mathbf{N}_{(k)}(U)=w_{k \zeta}^{\xi} U_{X}^{\zeta} \mathbf{e}_{(\xi)}(U),
$$

we have from (2.15)

$$
\left(q_{k}^{\tau}\right)_{X}=\left(N_{(k)}^{\tau}\right)_{X}, \quad\left(p_{k}^{l}\right)_{X}=\left(N_{(k)}^{N+l}\right)_{X}, \quad \tau=1, \ldots, N, l=1, \ldots g
$$

for the components of $\mathbf{N}_{(k)}(U)$.

We normalize the functions $q_{k}^{\top}(U)$ and $p_{k}^{l}(U)$ such that

$$
q_{k}^{\top}(z)=0, \quad p_{k}^{l}(z)=0 .
$$

We then have

$$
q_{k}^{\tau}(U)=N_{(k)}^{\tau}(U), \quad p_{k}^{l}(U)=N_{(k)}^{N+l}(U)+e_{k} \delta_{k}^{l}
$$

Now, using the equalities

$$
\left(q_{k}^{\tau}\right)_{X}=\frac{d}{d X} q_{k}^{\tau}-q_{k}^{\tau} \frac{d}{d X}, \quad\left(p_{k}^{l}\right)_{X}=\frac{d}{d X} p_{k}^{l}-p_{k}^{l} \frac{d}{d X}
$$

on $L\left(\mathcal{M}^{N}, z\right)$, we can write

$$
\begin{aligned}
& \hat{J}_{F}^{\nu \xi} \hat{\Omega}_{\xi \mu} \mid{ }_{\gamma_{0}(z)}=\sum_{\tau=1}^{N} \epsilon^{\tau}\left[g^{\nu \xi}\left(\frac{\partial n^{\tau}}{\partial U^{\xi}}\right)_{X}+b_{\eta}^{\nu \xi} U_{X}^{\eta} \frac{\partial n^{\tau}}{\partial U^{\xi}}+\sum_{k=1}^{g} e_{k} w_{k \eta}^{v} U_{X}^{\eta} D^{-1} \frac{d}{d X} q_{k}^{\tau}\right] D^{-1} \frac{\partial n^{\tau}}{\partial U^{\mu}} \\
& +\sum_{l=1}^{g} e_{l}\left[g^{\nu \xi}\left(\frac{\partial h^{l}}{\partial U^{\xi}}\right)_{X}+b_{\eta}^{\nu \xi} U_{X}^{\eta} \frac{\partial h^{l}}{\partial U^{\xi}}+\sum_{k=1}^{g} e_{k} w_{k \eta}^{v} U_{X}^{\eta} D^{-1} \frac{d}{d X} p_{k}^{l}\right] D^{-1} \frac{\partial h^{l}}{\partial U^{\mu}} \\
& +g^{\nu \xi}\left[\sum_{\tau=1}^{N} \epsilon^{\tau} \frac{\partial n^{\tau}}{\partial U^{\xi}} \frac{d}{d X} D^{-1} \frac{\partial n^{\tau}}{\partial U^{\mu}}+\sum_{l=1}^{g} e_{l} \frac{\partial h^{l}}{\partial U^{\xi}} \frac{d}{d X} D^{-1} \frac{\partial h^{l}}{\partial U^{\mu}}\right] \\
& -\sum_{k=1}^{g} \sum_{\tau=1}^{N} e_{k} \epsilon^{\tau} w_{k \eta}^{v} U_{X}^{\eta} D^{-1} q_{k}^{\tau} \frac{d}{d X} D^{-1} \frac{\partial n^{\top}}{\partial U^{\mu}} \\
& -\sum_{k=1}^{g} \sum_{l=1}^{g} e_{k} e_{l} w_{k \eta}^{v} U_{X}^{\eta} D^{-1} p_{k}^{l} \frac{d}{d X} D^{-1} \frac{\partial h^{l}}{\partial U^{\mu}} \text {. }
\end{aligned}
$$

We can here replace $(d / d X) D^{-1}$ by identity, and we have

$$
\left(D^{-1} f_{X}\right)=f(X)-\frac{1}{2}[f(-\infty)+f(+\infty)]
$$

for any function $f(U)$ on $L\left(M^{N}, z\right)$ according to the definition of $D^{-1}$. So, according to normalization (2.19), we can replace the operators $D^{-1}(d / d X) q_{k}^{\tau}$ and $D^{-1}(d / d X) p_{k}^{l}$ just by $q_{k}^{\top}$ and $p_{k}^{l}$ on $L\left(\mathcal{M}^{N}, z\right)$. 
According to the same normalization, the expressions within the brackets in the first two terms are equal to

$$
\left[\hat{J}_{F}^{\nu \xi} \frac{\partial n^{\tau}}{\partial U^{\xi}}\right]_{L\left(\mu^{N}, z\right)}=0, \quad\left[\hat{J}_{F}^{\nu \xi} \frac{\partial h^{l}}{\partial U^{\xi}}\right]_{L\left(\mu^{N}, z\right)}=w_{l \eta}^{v} U_{X}^{\eta}
$$

So, we have

$$
\begin{aligned}
\left.\hat{J}_{F}^{\nu \xi} \hat{\Omega}_{\xi \mu}\right|_{V_{0}(z)}= & \sum_{l=1}^{g} e_{l} w_{l \eta}^{\nu} U_{x}^{\eta} D^{-1} \frac{\partial h^{l}}{\partial U^{\mu}}+g^{\nu \xi}\left[\sum_{\tau=1}^{N} \epsilon^{\tau} \frac{\partial n^{\tau}}{\partial U^{\xi}} \frac{\partial n^{\tau}}{\partial U^{\mu}}+\sum_{l=1}^{g} e_{l} \frac{\partial h^{l}}{\partial U^{\xi}} \frac{\partial h^{l}}{\partial U^{\mu}}\right] \\
& -\sum_{k=1}^{g} e_{k} w_{k \eta}^{v} U_{X}^{\eta} D^{-1}\left[\sum_{\tau=1}^{N} \epsilon^{\tau} q_{k}^{\tau} \frac{\partial n^{\tau}}{\partial U^{\mu}}+\sum_{l=1}^{g} e_{l} p_{k}^{l} \frac{\partial h^{l}}{\partial U^{\mu}}\right] .
\end{aligned}
$$

Using (2.20) and (2.16), we now get

$$
\sum_{\tau=1}^{N} \epsilon^{\tau} q_{k}^{\tau} \frac{\partial n^{\tau}}{\partial U^{\mu}}+\sum_{l=1}^{g} e_{l} p_{k}^{l} \frac{\partial h^{l}}{\partial U^{\mu}}=\left\langle\mathbf{N}_{(k)}, \mathbf{e}_{(\mu)}\right\rangle+\frac{\partial h^{k}}{\partial U^{\mu}}=\frac{\partial h^{k}}{\partial U^{\mu}}
$$

Also, using the evident relation

$$
\sum_{\tau=1}^{N} \epsilon^{\tau} \frac{\partial n^{\tau}}{\partial U^{\xi}} \frac{\partial n^{\tau}}{\partial U^{\mu}}+\sum_{l=1}^{g} e_{l} \frac{\partial h^{l}}{\partial U^{\xi}} \frac{\partial h^{l}}{\partial U^{\mu}} \equiv g_{\xi \mu}(U),
$$

we get the statement (I) of the theorem.

(II) We have

$$
\begin{aligned}
\hat{\Omega}_{v \xi} \hat{J}_{F}^{\xi \mu}= & \left(\sum_{\tau=1}^{N} \epsilon^{\tau} \frac{\partial n^{\tau}}{\partial U^{v}} D^{-1} \frac{\partial n^{\tau}}{\partial U^{\xi}}+\sum_{l=1}^{g} e_{l} \frac{\partial h^{l}}{\partial U^{v}} D^{-1} \frac{\partial h^{l}}{\partial U^{\xi}}\right) \\
& \times\left(g^{\xi \mu} \frac{d}{d X}+b_{\eta}^{\xi \mu} U_{X}^{\eta}+\sum_{k=1}^{g} e_{k} w_{k \eta}^{\xi} U_{X}^{\eta} D^{-1} w_{k \zeta}^{\mu} U_{X}^{\zeta}\right) \\
= & \sum_{\tau=1}^{N} \epsilon^{\tau} \frac{\partial n^{\tau}}{\partial U^{v}} D^{-1} \frac{d}{d X} \frac{\partial n^{\tau}}{\partial U^{\xi}} g^{\xi \mu}+\sum_{l=1}^{g} \frac{\partial h^{l}}{\partial U^{v}} D^{-1} \frac{d}{d X} \frac{\partial h^{l}}{\partial U^{\xi}} g^{\xi \mu} \\
& -\sum_{\tau=1}^{N} \epsilon^{\tau} \frac{\partial n^{\tau}}{\partial U^{v}} D^{-1}\left(\frac{\partial n^{\tau}}{\partial U^{\xi}} g^{\xi \mu}\right)_{X}-\sum_{l=1}^{g} \frac{\partial h^{l}}{\partial U^{v}} D^{-1}\left(\frac{\partial h^{l}}{\partial U^{\xi}} g^{\xi \mu}\right)_{X} \\
& +\sum_{\tau=1}^{N} \epsilon^{\tau} \frac{\partial n^{\tau}}{\partial U^{v}} D^{-1} \frac{\partial n^{\tau}}{\partial U^{\xi}} b_{\eta}^{\xi \mu} U_{X}^{\eta}+\sum_{l=1}^{g} e_{l} \frac{\partial h^{l}}{\partial U^{v}} D^{-1} \frac{\partial h^{l}}{\partial U^{\xi}} b_{\eta}^{\xi \mu} U_{X}^{\eta} \\
& +\sum_{\tau=1}^{N} \sum_{k=1}^{g} \epsilon^{\tau} e_{k} \frac{\partial n^{\tau}}{\partial U^{v}} D^{-1}\left(\frac{d}{d X} q_{k}^{\tau}-q_{k}^{\tau} \frac{d}{d X}\right) D^{-1} w_{k \zeta}^{\mu} U_{X}^{\zeta} \\
& +\sum_{l=1}^{g} \sum_{k=1}^{g} e_{l} e_{k} \frac{\partial h^{l}}{\partial U^{v}} D^{-1}\left(\frac{d}{d X} p_{k}^{l}-p_{k}^{l} \frac{d}{d X}\right) D^{-1} w_{k \zeta}^{\mu} U_{X}^{\zeta} .
\end{aligned}
$$

Again, we can replace the operators $(d / d X) D^{-1}$ by identity and $D^{-1}(d / d X) q_{k}^{\tau}$ and $D^{-1}(d / d X) p_{k}^{l}$ by $q_{k}^{\tau}$ and $p_{k}^{l}$. Then, according to the definition of coordinates $h^{l}$, we 
have $\left(\partial h^{l} / \partial U^{\xi}\right)(z)=0$; so, we also put $D^{-1}(d / d X)\left(\partial h^{l} / \partial U^{\xi}\right)=\left(\partial h^{l} / \partial U^{\xi}\right)$. Now, using the same arguments, We get

$$
\begin{aligned}
\hat{\Omega}_{\nu \xi} \hat{J}_{F}^{\xi \mu}= & \hat{I}+\sum_{\tau=1}^{N} \epsilon^{\tau} \frac{\partial n^{\tau}}{\partial U^{v}}\left[D^{-1} \frac{d}{d X}-\hat{I}\right] \frac{\partial n^{\tau}}{\partial U^{\xi}} g^{\xi \mu} \\
& -\sum_{\tau=1}^{N} \epsilon^{\tau} \frac{\partial n^{\tau}}{\partial U^{v}} D^{-1}\left[\hat{J}_{F}^{\mu \xi} \frac{\partial n^{\tau}}{\partial U^{\xi}}\right]_{L^{\left(\mu^{N}, z\right)}}-\sum_{l=1}^{g} e_{l} \frac{\partial h^{l}}{\partial U^{v}} D^{-1}\left[\hat{J}_{F}^{\mu \xi} \frac{\partial h^{l}}{\partial U^{\xi}}\right]_{L\left(\mu^{N}, z\right)} \\
& +\sum_{k=1}^{g}\left(\sum_{\tau=1}^{N} \epsilon^{\tau} \frac{\partial n^{\tau}}{\partial U^{\nu}} q_{k}^{\tau} D^{-1} w_{k \zeta}^{\mu} U_{X}^{\zeta}+\sum_{l=1}^{g} e_{l} \frac{\partial h^{l}}{\partial U^{\nu}} p_{k}^{l} D^{-1} w_{k \zeta}^{\mu} U_{X}^{\zeta}\right)
\end{aligned}
$$

(we used the skew-symmetry of the operator $\hat{J}_{F}^{\mu \xi}$ for the action from the right).

Again, using (2.24) and (2.26), we get

$$
\hat{\Omega}_{\nu \xi} \hat{J}_{F}^{\xi \mu}=\hat{I}+\sum_{\tau=1}^{N} \epsilon^{\tau} \frac{\partial n^{\tau}}{\partial U^{v}}\left[D^{-1} \frac{d}{d X}-\hat{I}\right] \frac{\partial n^{\tau}}{\partial U^{\xi}} g^{\xi \mu},
$$

that is,

$$
\hat{\Omega}_{\nu \xi} \hat{J}_{F}^{\xi \mu} f_{\mu}(X)=f_{\mu}(X)-\sum_{\tau=1}^{N} \epsilon^{\tau} \frac{\partial n^{\tau}}{\partial U^{v}}(X) \frac{\partial n^{\tau}}{\partial U^{\xi}}(z) g^{\xi \mu}(z) f_{\mu}(z)
$$

for any $f_{\mu}(X)$. From the definition of $\mathscr{F}_{0}(z)$, we now obtain the part (II) of the theorem.

REMARK 2.3. We can also say that the operator $\hat{J}_{F}^{v \xi} \hat{\Omega}_{\xi \mu}$ is identity if it is acting from the left on $\mathscr{F}_{0}(z)$ and from the right on $\mathscr{V}_{0}(z)$. This interpretation will be convenient later for the consideration of the recursion operator.

We also introduce the momentum functional $P$ generating the flow

$$
U_{T}^{v}=U_{X}^{v}
$$

with respect to the general bracket (1.17) (with nondegenerate $g^{v \mu}(U)$ ).

LEMMA 2.4. Any F-bracket (1.17) with nondegenerate tensor $g^{v \mu}(U)$ has the local momentum operator $P$ generating the flow (2.32) on the space $L\left(M^{N}, z\right)$. The functional $P$ can be written in the form

$$
P=\int_{-\infty}^{+\infty} p(U) d X=\frac{1}{2} \int_{-\infty}^{+\infty}\left(\sum_{\tau=1}^{N} \epsilon^{\tau} n^{\top} n^{\top}+\sum_{k=1}^{g} e_{k} h^{k} h^{k}\right) d X,
$$

where the functions $n^{T}$ and $h^{k}$ correspond to the loop space $L\left(M^{N}, z\right)$.

Proof. We should here just prove the relation

$$
\frac{\partial p}{\partial U^{v}}=\hat{\Omega}_{\nu \xi} U_{X}^{\xi}
$$


on $L\left(\mu^{N}, z\right)$ according to part (I) of Theorem 2.2. So, we have

$$
\begin{aligned}
\sum_{\tau=1}^{N} \epsilon^{\tau} \frac{\partial n^{\tau}}{\partial U^{v}} D^{-1} & \frac{\partial n^{\tau}}{\partial U^{\xi}} U_{X}^{\xi}+\sum_{k=1}^{g} e_{k} \frac{\partial h^{k}}{\partial U^{v}} D^{-1} \frac{\partial h^{k}}{\partial U^{\xi}} U_{X}^{\xi} \\
& =\sum_{\tau=1}^{N} \epsilon^{\tau} \frac{\partial n^{\tau}}{\partial U^{v}} n^{\tau}+\sum_{k=1}^{g} e_{k} \frac{\partial h^{k}}{\partial U^{v}} h^{k}=\frac{\partial}{\partial U^{v}} p
\end{aligned}
$$

3. The $\lambda$-pencils and the integrable hierarchies. We now consider the operator

$$
\begin{aligned}
\hat{J}_{\lambda}^{v \mu}= & \hat{J}_{(0)}^{v \mu}+\lambda \hat{J}_{(1)}^{v \mu}=\left(g_{(0)}^{v \mu}+\lambda g_{(1)}^{v \mu}\right) \frac{d}{d X}+\left(b_{(0) \eta}^{v \mu}+\lambda b_{(1) \eta}^{v \mu}\right) U_{X}^{\eta} \\
& +\sum_{k=1}^{g_{0}} e_{(0) k} w_{(0) k \eta}^{v} U_{X}^{\eta} D^{-1} w_{(0) k \zeta}^{\mu} U_{X}^{\zeta}+\lambda \sum_{k=1}^{g_{1}} e_{(1) k} w_{(1) k \eta}^{v} U_{X}^{\eta} D^{-1} w_{(1) k \zeta}^{\mu} U_{X}^{\zeta}
\end{aligned}
$$

for $\hat{J}_{(0)}^{v \mu}$ and $\hat{J}_{(1)}^{v \mu}$ having form (1.18). We will call the $\lambda$-pencil (3.1) nondegenerate, for small $\lambda$, if $\operatorname{det} g_{(0)}^{v \mu}(U) \neq 0$.

We will admit here that the linear spaces $\mathcal{W}_{(0)}$ and $\mathcal{W}_{(1)}$, generated by the sets

$$
\left\{w_{(0) 1 \eta}^{v} U_{X}^{\eta}, \ldots, w_{(0) g_{0} \eta}^{v} U_{X}^{\eta}\right\}, \quad\left\{w_{(1) 1 \eta}^{v} U_{X}^{\eta}, \ldots, w_{(1) g_{1} \eta}^{v} U_{X}^{\eta}\right\},
$$

can have a nontrivial intersection $\mathscr{V}$.

We introduce the basis in $\mathscr{V}$

$$
\left\{\hat{v}_{1 \eta}^{v} U_{X}^{\eta}, \ldots, \hat{v}_{d \eta}^{v} U_{X}^{\eta}\right\}
$$

where $d=\operatorname{dim} \mathcal{V}$, and consider the linear space $\mathcal{W}$, generated by all the flows from $\mathcal{W}_{(0)}$ and $\mathcal{W}_{(1)}\left(\operatorname{dim} \mathcal{W}=g_{0}+g_{1}-d\right)$ with basis

$$
\begin{aligned}
\mathscr{B} & =\left\{\hat{w}_{(0) 1 \eta}^{v} U_{X}^{\eta}, \ldots, \hat{w}_{(0)\left(g_{0}-d\right) \eta}^{v} U_{X}^{\eta}, \hat{v}_{1 \eta}^{v} U_{X}^{\eta}, \ldots, \hat{v}_{d \eta}^{v} U_{X}^{\eta}, \hat{w}_{(1) 1 \eta}^{v} U_{X}^{\eta}, \ldots, \hat{w}_{(1)\left(g_{1}-d\right) \eta}^{v} U_{X}^{\eta}\right\} \\
& =\left\{\tilde{w}_{1 \eta}^{v}(U) U_{X}^{\eta}, \ldots, \tilde{w}_{g_{0}+g_{1}-d, \eta}^{v}(U) U_{X}^{\eta}\right\},
\end{aligned}
$$

where $\hat{w}_{(0) k \eta}^{v}$ and $\hat{w}_{(1) s \eta}^{v}$ are some linear combinations of operators $w_{(0)}$ and $w_{(1)}$, respectively, and the flows corresponding to $\hat{w}_{(0) k}, \hat{v}_{m}$, and $\hat{w}_{(1) s}$ are all linearly independent.

The flows

$$
\begin{aligned}
& \left\{\hat{w}_{(0) 1 \eta}^{v} U_{X}^{\eta}, \ldots, \hat{w}_{(0)\left(g_{0}-d\right) \eta}^{v} U_{X}^{\eta}, \hat{v}_{1 \eta}^{v} U_{X}^{\eta}, \ldots, \hat{v}_{d \eta}^{v} U_{X}^{\eta}\right\}, \\
& \left\{\hat{v}_{1 \eta}^{v} U_{X}^{\eta}, \ldots, \hat{v}_{d \eta}^{v} U_{X}^{\eta}, \hat{w}_{(1) 1 \eta}^{v} U_{X}^{\eta}, \ldots, \hat{w}_{(1)\left(g_{1}-d\right) \eta}^{v} U_{X}^{\eta}\right\}
\end{aligned}
$$

will then give bases in $\mathcal{W}_{(0)}$ and $\mathscr{W}_{(1)}$, respectively.

The nonlocal part of the bracket $\hat{J}_{\lambda}^{v \mu}$

$$
\sum_{k=1}^{g_{0}} e_{(0) k} w_{(0) k \eta}^{\nu} U_{X}^{\eta} D^{-1} w_{(0) k \zeta}^{\mu} U_{X}^{\zeta}+\lambda \sum_{s=1}^{g_{1}} e_{(1) s} w_{(1) s \eta}^{\nu} U_{X}^{\eta} D^{-1} w_{(1) s \zeta}^{\mu} U_{X}^{\zeta}
$$

will correspond in our case to some quadratic form $Q_{\lambda}^{k s}$ (linear in $\lambda$ ), $k, s=1, \ldots, g_{0}+$ $g_{1}-d$ on the space $\mathcal{W}$. 
In our further consideration, the question if $Q_{\lambda}^{k s}$ is nondegenerate on $\mathcal{W}$ for $\lambda \neq 0$ or not will be important, and we will mainly consider the pencils (3.1) such that $Q_{\lambda}^{k s}$ is nondegenerate on $\mathcal{W}$.

We now formulate the properties of nondegenerate pencils (3.1) also satisfying the requirement of nondegeneracy of the form $Q_{\lambda}^{k s}$ for $\lambda \neq 0$ connected with the "canonical" integrable hierarchies.

THEOREM 3.1. Consider the nondegenerate pencil (3.1) $\left(\operatorname{det} g_{(0)}^{v \mu} \neq 0\right)$ such that the form $Q_{\lambda}^{k s}$ is also nondegenerate on $\mathcal{W}$ for $\lambda \neq 0$ (small enough). Then,

(I) it is possible to introduce the local functionals

$$
\begin{aligned}
N^{v}(\lambda) & =\int_{-\infty}^{+\infty} n^{v}(U, \lambda) d X, \quad v=1, \ldots, N, \\
P(\lambda) & =\int_{-\infty}^{+\infty} p(U, \lambda) d X, \\
H_{(0)}^{k}(\lambda) & =\int_{-\infty}^{+\infty} h_{(0)}^{k}(U, \lambda) d X, \quad k=1, \ldots, g_{0}, \\
H_{(1)}^{s}(\lambda) & =\int_{-\infty}^{+\infty} h_{(1)}^{s}(U, \lambda) d X, \quad s=1, \ldots, g_{1},
\end{aligned}
$$

which are the Casimirs, momentum operator and the Hamiltonian functions for the flows $w_{(0) k \eta}^{v}(U) U_{X}^{\eta}$ and $w_{(1) s \eta}^{v}(U) U_{X}^{\eta}$ for the bracket $\hat{J}_{\lambda}^{v \mu}$, respectively;

(II) all the functions $n^{v}(U, \lambda), p(U, \lambda), h_{(0)}^{k}(U, \lambda)$, and $h_{(1)}^{s}(U, \lambda)$ are regular at $\lambda \rightarrow$ 0 and can be represented as regular series

$$
\begin{aligned}
n^{v}(U, \lambda) & =\sum_{q=0}^{+\infty} n_{q}^{v}(U) \lambda^{q}, & p(U, \lambda) & =\sum_{q=0}^{+\infty} p_{q}(U) \lambda^{q}, \\
h_{(0)}^{k}(U, \lambda) & =\sum_{q=0}^{+\infty} h_{(0), q}^{k}(U) \lambda^{q}, & h_{(1)}^{s}(U, \lambda) & =\sum_{q=0}^{+\infty} h_{(1), q}^{s}(U) \lambda^{q} .
\end{aligned}
$$

Moreover, we can choose these functionals in such a way that

$$
\begin{array}{cl}
\frac{\partial p_{q}}{\partial U^{\mu}}(z)=0, \quad \frac{\partial h_{(0), q}^{k}}{\partial U^{\mu}}(z)=0, & \frac{\partial h_{(1), q}^{s}}{\partial U^{\mu}}(z)=0, \quad q \geq 0, \\
\frac{\partial n_{q}^{v}}{\partial U^{\mu}}(z)=0, \quad q \geq 1, & \frac{\partial n_{0}^{v}}{\partial U^{\mu}}(z)=e_{\mu}^{v},
\end{array}
$$

where det $e_{\mu}^{v} \neq 0$;

(III) the integrals $N^{v}(0), P(0), H_{(0)}^{k}(0)$, and $H_{(1)}^{s}(0)$ are the Casimirs, momentum operator, and the Hamiltonian functions for the flows $w_{(0) k \eta}^{v}(U) U_{X}^{\eta}$ and $w_{(1) s \eta}^{v}(U) U_{X}^{\eta}$ with respect to $\hat{J}_{(0)}^{v \mu}$, while the flows generated by the functionals

$$
F_{q}=\int_{-\infty}^{+\infty} f_{q}(U) d X
$$

are connected by the relation

$$
\hat{J}_{(0)}^{v \xi} \frac{\partial f_{(q+1)}}{\partial U \xi}=-\hat{J}_{(1)}^{v \xi} \frac{\partial f_{q}}{\partial U \xi}
$$


for any functional $F(\lambda)$ from the set (3.7). All the functionals $F_{q}$ given by the expansions (3.8) generate the local flows and commute with each other with respect to both brackets $\hat{J}_{(0)}$ and $\hat{J}_{(1)}$.

Proof. We now put $\lambda>0$ and write $\hat{J}_{\lambda}^{v \mu}$ in the form

$$
\begin{aligned}
\hat{J}_{\lambda}^{v \mu}= & \left(g_{(0)}^{v \mu}+\lambda g_{(1)}^{v \mu}\right) \frac{d}{d X}+\left(b_{(0) \eta}^{v \mu}+\lambda b_{(1) \eta}^{v \mu}\right) U_{X}^{\eta} \\
& +\sum_{k=1}^{g_{0}} e_{(0) k} w_{(0) k \eta}^{v} U_{X}^{\eta} D^{-1} w_{(0) k \zeta}^{\mu} U_{X}^{\zeta} \\
& +\sum_{k=1}^{g_{1}} e_{(1) k} \sqrt{\lambda} w_{(1) k \eta}^{v} U_{X}^{\eta} D^{-1} \sqrt{\lambda} w_{(1) k \zeta}^{\mu} U_{X}^{\zeta} .
\end{aligned}
$$

In the case of the nondegenerate form $Q_{\lambda}^{k s}$ on $\mathcal{W}$, we can consider the corresponding embedding of $\mathcal{M}^{N}$ to $\mathbb{R}^{N+g_{0}+g_{1}}$ depending on $\lambda$. Indeed, all the flows $\tilde{w}_{s}$ from the set $\mathscr{B}$ will in this case satisfy conditions

$$
\begin{gathered}
g_{\lambda}^{v \xi} \tilde{w}_{s \xi}^{\mu}=g_{\lambda}^{\mu \xi} \tilde{w}_{s \xi}^{v}, \quad \nabla_{\nu} \tilde{w}_{s \eta}^{\mu}=\nabla_{\eta} \tilde{w}_{s v}^{\mu}, \\
R_{\eta \zeta}^{v \mu}=\sum_{k, s}\left(\tilde{w}_{k \eta}^{v} Q_{\lambda}^{k s} \tilde{w}_{s \zeta}^{\mu}-\tilde{w}_{k \eta}^{\mu} Q_{\lambda}^{k s} \tilde{w}_{s \zeta}^{v}\right)
\end{gathered}
$$

for nondegenerate $g_{\lambda}^{\mu \xi}$ according to Ferapontov theorem.

Since the operators $w_{(0) k \eta}^{v}(U)$ and $w_{(1) s \eta}^{v}(U)$ are just linear combinations of $\tilde{w}_{n \eta}^{v}(U)$ (with constant coefficients) and the form $Q_{\lambda}^{k s}$ coincides with the nonlocal part of (3.12), we will have the corresponding Gauss and Petersson-Codazzi equations for these flows and the curvature tensor $R_{\eta \zeta}^{v \mu}$. So, for nondegenerate $g_{\lambda}^{\nu \mu}$, we will get the local embedding of $\mu^{N}$ to $\mathbb{R}^{N+g_{0}+g_{1}}$ (actually, to some subspace $\mathbb{R}^{N+g_{0}+g_{1}-d} \subset \mathbb{R}^{N+g_{0}+g_{1}}$ ) depending on $\lambda$.

Since the embedding is defined up to the Poincaré transformation in $\mathbb{R}^{N+g_{0}+g_{1}}$, we can choose, at all $\lambda$, the coordinates $Z^{I}, I=1, \ldots, N+g_{0}+g_{1}$ in such a way that

(1) all $Z^{I}=0$ at the point $z \in \mathcal{M}^{N}$, and the metric $G^{I J}$ in $\mathbb{R}^{N+g_{0}+g_{1}}$ has the form

$$
G^{I J}=E^{I} \delta^{I J}
$$

where $E^{N+k}=e_{(0) k}, k=1, \ldots, g_{0}, E^{N+g_{0}+s}=e_{(1) s}, s=1, \ldots, g_{1} ;$

(2) the first $N$ coordinates $Z^{v}, v=1, \ldots, N$ are tangential to $\mu^{N}$ at the point $z \in M^{N}$ at all $\lambda$;

(3) the last $g_{0}+g_{1}$ coordinates are orthogonal to $\mathcal{M}^{N}$ at the point $z$, and the Weingarten operators

$$
w_{(0) 1 \eta}^{v}(U), \ldots, w_{(0) g_{0} \eta}^{v}(U), \sqrt{\lambda} w_{(1) 1 \eta}^{v}(U), \ldots, \sqrt{\lambda} w_{(1) g_{1} \eta}^{v}(U)
$$

correspond to the parallel vector fields $\mathbf{N}_{(0)(k)}(U), \mathbf{N}_{(1)(s)}(U)$ in the normal bundle such that

$$
\mathbf{N}_{(0)(k)}(z)=\left(0, \ldots, 0,-E^{N+k}, 0, \ldots, 0\right)^{T}
$$


$\left(-E^{N+k}\right.$ stays at the position $\left.N+k\right) k=1, \ldots, g_{0}$,

$$
\mathbf{N}_{(1)(k)}(z)=\left(0, \ldots, 0,-E^{N+g_{0}+s}, 0, \ldots, 0\right)^{T}
$$

$\left(-E^{N+g_{0}+s}\right.$ stays at the position $\left.N+g_{0}+s\right) s=1, \ldots, g_{1}$.

So, according to [25], the restriction of the first $N$ coordinates $Z^{1}, \ldots, Z^{N}$ gives the Casimirs of bracket (3.12) on $L\left(\mu^{N}, z\right)$

$$
\tilde{N}^{v}(\lambda)=\int_{-\infty}^{+\infty} \tilde{n}^{v}(U, \lambda) d X=\left.\int_{-\infty}^{+\infty} Z^{v}\right|_{\mathcal{M}^{N}}(U, \lambda) d X, \quad v=1, \ldots, N
$$

while the restrictions of the last $g_{0}+g_{1}$ coordinates give the Hamiltonian functions for the flows $w_{(0) k \eta}^{v} U_{X}^{\eta}$

$$
\tilde{H}_{(0)}^{k}(\lambda)=\int_{-\infty}^{+\infty} \tilde{h}_{(0)}^{k}(U, \lambda) d X=\left.\int_{-\infty}^{+\infty} Z^{N+k}\right|_{\mathcal{M}^{N}}(U, \lambda) d X, \quad k=1, \ldots, g_{0}
$$

and $\sqrt{\lambda} w_{(1) s \eta}^{v} U_{X}^{\eta}$

$$
\tilde{H}_{(1)}^{s}(\lambda)=\int_{-\infty}^{+\infty} \tilde{h}_{(1)}^{s}(U, \lambda) d X=\left.\int_{-\infty}^{+\infty} Z^{N+g_{0}+s}\right|_{\mathcal{M}^{N}}(U, \lambda) d X, \quad s=1, \ldots, g_{1} .
$$

REMARK 3.2. For $\lambda<0$, the signature of $\mathbb{R}^{N+g_{0}+g_{1}}$ may be different from the case $\lambda>0$, but all the statements of the theorem will certainly be also true.

We now study the $\lambda$-dependence of the functions $\tilde{n}^{v}(U, \lambda), \tilde{h}_{(0)}^{k}(U, \lambda)$, and $\tilde{h}_{(1)}^{s}(U, \lambda)$ at $\lambda \rightarrow 0$.

Consider $N$ tangential vectors to $\mathcal{M}^{N}$, corresponding to the coordinate system $\left\{U^{v}\right\}$, that is,

$$
\mathbf{e}_{(v)}=\left(\frac{\partial Z^{1}}{\partial U^{v}}, \ldots, \frac{\partial Z^{N+g_{0}+g_{1}}}{\partial U^{v}}\right)^{T}, \quad v=1, \ldots, N
$$

We have the following relations (in $\left.\mathbb{R}^{N+g_{0}+g_{1}}\right)$ for the differentials of $\mathbf{e}_{(v)}(U, \lambda)$, $\mathbf{N}_{(0)(k)}(U, \lambda)$, and $\mathbf{N}_{(1)(s)}(U, \lambda)$ on $\mathcal{M}^{N}$ :

$$
\begin{aligned}
d \mathbf{e}_{(v)}= & \Gamma_{v \eta}^{\mu}(U, \lambda) \mathbf{e}_{(\mu)} d U^{\eta}-\sum_{k=1}^{g_{0}} E^{N+k} g_{v \mu}(U, \lambda) w_{(0) k \eta}^{\mu}(U, \lambda) \mathbf{N}_{(0)(k)} d U^{\eta} \\
& -\sqrt{\lambda} \sum_{s=1}^{g_{1}} E^{N+g_{0}+s} g_{v \mu}(U, \lambda) w_{(1) s \eta}^{\mu}(U, \lambda) \mathbf{N}_{(1)(s)} d U^{\eta} \\
d \mathbf{N}_{(0)(k)}= & w_{(0) k \eta}^{v}(U, \lambda) \mathbf{e}_{(v)} d U^{\eta} \\
d \mathbf{N}_{(1)(s)}= & \sqrt{\lambda} w_{(1) s \eta}^{v}(U, \lambda) \mathbf{e}_{(v)} d U^{\eta}
\end{aligned}
$$

where $\Gamma_{v \eta}^{\mu}=-g_{v \xi} b_{\eta}^{\xi \mu}, g_{v \xi} g^{\xi \mu} \equiv \delta_{v}^{\mu}$. 
So, for any curve $\gamma(t)$ on $\mu^{N}(\gamma(0)=z)$, we have the evolution system for $\mathbf{e}_{(v)}(t)$, $\mathbf{N}_{(0)(k)}(t)$, and $\mathbf{N}_{(1)(s)}(t)$ having the general form

$$
\frac{d}{d t}\left(\begin{array}{c}
\mathbf{e}_{(v)}(t) \\
\mathbf{N}_{(0)(k)}(t) \\
\mathbf{N}_{(1)(s)}(t)
\end{array}\right)=\left(\begin{array}{ccc}
*(t, \lambda) & *(t, \lambda) & \sqrt{\lambda} *(t, \lambda) \\
*(t, \lambda) & 0 & 0 \\
\sqrt{\lambda} *(t, \lambda) & 0 & 0
\end{array}\right)\left(\begin{array}{c}
\mathbf{e}_{(v)}(t) \\
\mathbf{N}_{(0)(k)}(t) \\
\mathbf{N}_{(1)(s)}(t)
\end{array}\right)
$$

where all $*(t, \lambda)$ are regular at $\lambda \rightarrow 0$ (matrix-) functions of $\lambda$.

The formal solution of (3.23) can be written as the chronological exponent

$$
T \exp \left(\int_{0}^{t} \hat{H}(t) d t\right)=\hat{I}+\sum_{n=1}^{+\infty} \frac{1}{n !} \int_{0}^{t_{1}} \cdots \int_{0}^{t_{n}} T\left(\hat{H}\left(t_{1}\right) \cdots \hat{H}\left(t_{n}\right)\right) d t_{1} \cdots d t_{n}
$$

applied to the initial data $\mathbf{e}_{(v)}(0), \mathbf{N}_{(0)(k)}(0)$, and $\mathbf{N}_{(1)(s)}(0)$, where $\hat{H}(t)$ is the matrix of system (3.23).

It is now easy to verify that, for any $n \geq 1$, we have

$$
T\left(\hat{H}\left(t_{1}\right) \cdots \hat{H}\left(t_{n}\right)\right)=\left(\begin{array}{ccc}
*(t, \lambda) & *(t, \lambda) & \sqrt{\lambda} *(t, \lambda) \\
*(t, \lambda) & *(t, \lambda) & \sqrt{\lambda} *(t, \lambda) \\
\sqrt{\lambda} *(t, \lambda) & \sqrt{\lambda} *(t, \lambda) & \lambda *(t, \lambda)
\end{array}\right)
$$

where all $*(t, \lambda)$ are regular matrix-functions at $\lambda \rightarrow 0$.

For the densities of Casimirs (3.18) and Hamiltonian functions (3.19) and (3.20), we can now write the equations

$$
\begin{aligned}
\frac{d \tilde{n}^{v}(t, \lambda)}{d t} & =\left[U_{t}^{\mu} \mathbf{e}_{(\mu)}(t, \lambda)\right]^{v}=\epsilon^{v}\left\langle U_{t}^{\mu} \mathbf{e}_{(\mu)}(t, \lambda), \mathbf{e}_{v}(0)\right\rangle \\
\frac{d \tilde{h}_{(0)}^{k}(t, \lambda)}{d t} & =-\left\langle U_{t}^{\mu} \mathbf{e}_{(\mu)}(t, \lambda), \mathbf{N}_{(0)(k)}(0)\right\rangle \\
\frac{d \tilde{h}_{(1)}^{s}(t, \lambda)}{d t} & =-\left\langle U_{t}^{\mu} \mathbf{e}_{(\mu)}(t, \lambda), \mathbf{N}_{(1)(s)}(0)\right\rangle
\end{aligned}
$$

along the same curve $\gamma(t)$ on $\mu^{N}$. Since $\gamma(t)$ is just the arbitrary curve, we have

$$
\tilde{n}^{v}(U, \lambda)=*(U, \lambda), \quad \tilde{h}_{(0)}^{k}(U, \lambda)=*(U, \lambda), \quad \tilde{h}_{(1)}^{s}(U, \lambda)=\sqrt{\lambda} *(U, \lambda),
$$

where $*(U, \lambda)$ are regular at $\lambda \rightarrow 0$.

It is easy to see that expression (2.33) for the momentum operator is regular at $\lambda \rightarrow 0$ in this case, and we can put

$$
h_{(0)}^{k}(U, \lambda)=\tilde{h}_{(0)}^{k}(U, \lambda), \quad h_{(1)}^{s}(U, \lambda)=\frac{1}{\sqrt{\lambda}} \tilde{h}_{(1)}^{s}(U, \lambda)
$$

to be regular at $\lambda \rightarrow 0$ densities of Hamiltonian functions $H_{(0)}^{k}(\lambda)$ and $H_{(1)}^{s}(\lambda)$. 
According to the geometric construction, we have

$$
\left.\frac{\partial h_{(0)}^{k}(U, \lambda)}{\partial U^{\mu}}\right|_{z} \equiv 0,\left.\quad \frac{\partial h_{(1)}^{s}(U, \lambda)}{\partial U^{\mu}}\right|_{z} \equiv 0
$$

and also

$$
\left.\frac{\partial p(U, \lambda)}{\partial U^{\mu}}\right|_{z} \equiv 0
$$

Since the functions $\tilde{n}^{v}(U, \lambda)$ locally give the coordinate system on $\mathcal{M}^{N}$ at every $\lambda$, we have

$$
\operatorname{det}\left(\frac{\partial \tilde{n}^{v}}{\partial U^{\mu}}(z, \lambda)\right) \neq 0
$$

and we can put

$$
n^{v}(U, \lambda)=\frac{\partial \tilde{n}^{v}}{\partial U^{\xi}}(z, 0) \frac{\partial U^{\xi}}{\partial \tilde{n}^{\eta}}(z, \lambda) \tilde{n}^{\eta}(U, \lambda)
$$

such that

$$
\left.\frac{\partial n^{v}(U, \lambda)}{\partial U^{\mu}}\right|_{z} \equiv \frac{\partial n^{v}}{\partial U^{\mu}}(z, 0)=\left(\mathbf{e}_{(\mu)}\right)^{v}, \quad v=1, \ldots, N,
$$

where $\mathbf{e}_{(\mu)}$ are the tangent vectors at the point $z$ for $\lambda=0, n^{v}(U, 0)=\tilde{n}^{v}(U, 0)$. So, we get parts (I) and (II) of the theorem.

For part (III), we first prove here the following lemma.

LEMMA 3.3. Under the conditions formulated in Theorem 3.1 the functionals

$$
\begin{aligned}
N_{p}^{v} & =\int_{-\infty}^{+\infty} n_{p}^{v}(U) d X, & P_{q} & =\int_{-\infty}^{+\infty} p_{q}(U) d X, \\
H_{(0) l}^{k} & =\int_{-\infty}^{+\infty} h_{(0) l}^{k}(U) d X, & H_{(1) t}^{s} & =\int_{-\infty}^{+\infty} h_{(1) t}^{s}(U) d X,
\end{aligned}
$$

$p, q, l, t=0,1, \ldots$, generate the local flows with respect to both $\hat{J}_{(0)}$ and $\hat{J}_{(1)}$ and commute with the functionals $N_{0}^{\mu}, P_{0}, H_{(0) 0}^{m}$, and $H_{(1) 0}^{n}$ with respect to the bracket $\hat{J}_{(0)}$.

Proof. Since the functionals $N_{0}^{\mu}$ are the annihilators of $\hat{J}_{(0)}$, they commute with all other functionals with respect to $\hat{J}_{(0)}$ on $L\left(\mu^{N}, z\right)$. Also, from the translational invariance of all functionals $N_{p}^{v}, P_{q}, H_{(0) l}^{k}$, and $H_{(1) t}^{s}$, we get that they also commute with the momentum operator $P_{0}$ of $\hat{J}_{(0)}$ with respect to $\hat{J}_{(0)}$.

We then know that the functionals $N^{v}(\lambda), P(\lambda), H_{(0)}^{k}(\lambda)$, and $H_{(1)}^{s}(\lambda)$ generate the local flows with respect to $\hat{J}_{\lambda}$. In the case of nondegenerate form $Q_{\lambda}^{k s}$ (on $\mathcal{W}$ ), this means that they are the conservation laws for all the flows $\tilde{w}_{n \eta}^{v}(U) U_{X}^{\eta}$ introduced in (3.4). So, they are the conservation laws for the flows $w_{(0) k \eta}^{v}(U) U_{X}^{\eta}$ and $w_{(1) s \eta}^{v}(U) U_{X}^{\eta}$ and generate the local flows with respect to $\hat{J}_{(0)}$ and $\hat{J}_{(1)}$. Now, since the flows $w_{(0) m \eta}^{v}(U) U_{X}^{\eta}$ and $w_{(1) n \eta}^{v}(U) U_{X}^{\eta}$ are generated by the functionals $H_{(0) 0}^{m}$ and $H_{(1) 0}^{n}$ with respect to $\hat{J}_{(0)}$, we get that all $N_{p}^{v}, P_{q}, H_{(0) l}^{k}$, and $H_{(1) t}^{s}$ should commute with $H_{(0) 0}^{m}$ and $H_{(1) 0}^{n}$ with respect to $\hat{J}_{(0)}$ on $L\left(\mathcal{M}^{N}, z\right)$. 
For the commutativity of all $N_{p}^{v}, P_{q}, H_{(0) l}^{k}$, and $H_{(1) t}^{s}$ with respect to both brackets, we can now use just the common approach for the bi-Hamiltonian systems [20] writing

$$
\delta F_{q} \hat{J}_{(0)} \delta G_{k}=-\delta F_{q-1} \hat{J}_{(1)} \delta G_{k}=\delta F_{q-1} \hat{J}_{(0)} \delta G_{k+1}=\cdots=\delta F_{0} \hat{J}_{(0)} \delta G_{k+q}=0
$$

for any two functionals $F_{q}$ and $G_{k}$ from the set (3.34) (the same for the bracket $\hat{J}_{(1)}$ ).

REMARK 3.4. We point out here that the requirement of nondegeneracy of the form $Q_{\lambda}^{k s}$ on $\mathcal{W}$ is important, and, in general, Theorem 3.1 is not true without it. As example, we consider here the Poisson pencil $\hat{J}_{\lambda}=\hat{J}_{(0)}+\lambda \hat{J}_{(1)}$ where

$$
\begin{aligned}
\hat{J}_{(0)}= & \left(\begin{array}{cc}
1 & 0 \\
0 & -1
\end{array}\right) \frac{d}{d X}+\left(\begin{array}{cc}
U^{1} & U^{2} \\
-U^{2} & -U^{1}
\end{array}\right)\left(\begin{array}{l}
U_{X}^{1} \\
U_{X}^{2}
\end{array}\right) D^{-1}\left(\begin{array}{ll}
1 & 0 \\
0 & 1
\end{array}\right)\left(\begin{array}{l}
U_{X}^{1} \\
U_{X}^{2}
\end{array}\right) \\
& +\left(\begin{array}{ll}
1 & 0 \\
0 & 1
\end{array}\right)\left(\begin{array}{l}
U_{X}^{1} \\
U_{X}^{2}
\end{array}\right) D^{-1}\left(\begin{array}{cc}
U^{1} & U^{2} \\
-U^{2} & -U^{1}
\end{array}\right)\left(\begin{array}{l}
U_{X}^{1} \\
U_{X}^{2}
\end{array}\right), \\
\hat{J}_{(1)}= & \left(\begin{array}{ll}
0 & 1 \\
1 & 0
\end{array}\right) \frac{d}{d X}+\left(\begin{array}{cc}
0 & 0 \\
2 U^{1} & 0
\end{array}\right)\left(\begin{array}{c}
U_{X}^{1} \\
U_{X}^{2}
\end{array}\right) D^{-1}\left(\begin{array}{ll}
1 & 0 \\
0 & 1
\end{array}\right)\left(\begin{array}{l}
U_{X}^{1} \\
U_{X}^{2}
\end{array}\right) \\
& +\left(\begin{array}{ll}
1 & 0 \\
0 & 1
\end{array}\right)\left(\begin{array}{l}
U_{X}^{1} \\
U_{X}^{2}
\end{array}\right) D^{-1}\left(\begin{array}{cc}
0 & 0 \\
2 U^{1} & 0
\end{array}\right)\left(\begin{array}{l}
U_{X}^{1} \\
U_{X}^{2}
\end{array}\right) .
\end{aligned}
$$

We have here the three-dimensional space $\mathcal{W}$ generated by the flows

$$
\begin{gathered}
\tilde{w}_{1 \eta}^{v} U_{X}^{\eta}=\left(\begin{array}{cc}
U^{1} & U^{2} \\
-U^{2} & -U^{1}
\end{array}\right)\left(\begin{array}{c}
U_{X}^{1} \\
U_{X}^{2}
\end{array}\right), \quad \tilde{w}_{2 \eta}^{v} U_{X}^{\eta}=\left(\begin{array}{c}
U_{X}^{1} \\
U_{X}^{2}
\end{array}\right), \\
\tilde{w}_{3 \eta}^{v} U_{X}^{\eta}=\left(\begin{array}{cc}
0 & 0 \\
2 U^{1} & 0
\end{array}\right)\left(\begin{array}{l}
U_{X}^{1} \\
U_{X}^{2}
\end{array}\right) .
\end{gathered}
$$

Operator $\hat{J}_{\lambda}$ can be written as

$$
\begin{aligned}
\hat{J}_{\lambda}= & \left(\begin{array}{cc}
1 & \lambda \\
\lambda & -1
\end{array}\right) \frac{d}{d X}+\left(\begin{array}{cc}
U^{1} & U^{2} \\
-U^{2}+2 \lambda U^{1} & -U^{1}
\end{array}\right)\left(\begin{array}{c}
U_{X}^{1} \\
U_{X}^{2}
\end{array}\right) D^{-1}\left(\begin{array}{c}
U_{X}^{1} \\
U_{X}^{2}
\end{array}\right) \\
& +\left(\begin{array}{c}
U_{X}^{1} \\
U_{X}^{2}
\end{array}\right) D^{-1}\left(\begin{array}{cc}
U^{1} & U^{2} \\
-U^{2}+2 \lambda U^{1} & -U^{1}
\end{array}\right)\left(\begin{array}{l}
U_{X}^{1} \\
U_{X}^{2}
\end{array}\right),
\end{aligned}
$$

and the form

$$
Q_{\lambda}=\left(\begin{array}{lll}
0 & 1 & 0 \\
1 & 0 & \lambda \\
0 & \lambda & 0
\end{array}\right)
$$

is degenerate on $\mathscr{W}$.

The metric

$$
g_{\lambda}^{v \mu}(U)=\left(\begin{array}{cc}
1 & \lambda \\
\lambda & -1
\end{array}\right)
$$


here is just the flat metric on $\mathbb{R}^{2}$, $\operatorname{det} g_{\lambda}^{v \mu} \neq 0$, and it is easy to check the equations

$$
g_{\lambda}^{\nu \xi} W_{1 \xi}^{\mu}(\lambda)=g_{\lambda}^{\mu \xi} W_{1 \xi}^{\nu}(\lambda), \quad g_{\lambda}^{\nu \xi} W_{2 \xi}^{\mu}=g_{\lambda}^{\mu \xi} W_{2 \xi}^{\nu}
$$

where

$$
W_{1 \xi}^{\mu}(\lambda)=\left(\begin{array}{cc}
U^{1} & U^{2} \\
-U^{2}+2 \lambda U^{1} & -U^{1}
\end{array}\right), \quad W_{2 \xi}^{\mu}(\lambda)=\left(\begin{array}{ll}
1 & 0 \\
0 & 1
\end{array}\right)
$$

Also,

$$
\partial_{\nu} W_{1 \mu}^{\xi}(\lambda)=\partial_{\mu} W_{1 v}^{\xi}(\lambda), \quad \partial_{\nu} W_{2 \mu}^{\xi}=\partial_{\mu} W_{2 v}^{\xi}
$$

in the flat coordinates $U^{1}, U^{2}$, and

$$
\begin{aligned}
R_{12}^{12} & =W_{11}^{1}(\lambda) W_{22}^{2}+W_{21}^{1} W_{12}^{2}(\lambda)-W_{11}^{2}(\lambda) W_{22}^{1}-W_{21}^{2} W_{12}^{1}(\lambda) \\
& =W_{11}^{1}(\lambda)+W_{12}^{2}(\lambda) \equiv 0
\end{aligned}
$$

so, $\hat{J}_{\lambda}$ represents a Poisson bracket of Ferapontov type for all $\lambda$.

Both $g_{(0)}^{\nu \mu}$ and $g_{(1)}^{v \mu}$ are nondegenerate in this case. However, the flow $\tilde{w}_{1 \eta}^{v} U_{X}^{\eta}$ is not Hamiltonian with respect to $\hat{J}_{(1)}$, and $\tilde{w}_{3 \eta}^{v} U_{X}^{\eta}$ is not Hamiltonian with respect to $\hat{J}_{(0)}$ as follows from

$$
g_{(0)}^{\nu \xi} \tilde{w}_{3 \xi}^{\mu} \neq g_{(0)}^{\mu \xi} \tilde{w}_{3 \xi}^{\nu}, \quad g_{(1)}^{\nu \xi} \tilde{w}_{1 \xi}^{\mu} \neq g_{(1)}^{\mu \xi} \tilde{w}_{1 \xi}^{\nu}
$$

It is also easy to check that the flows $\tilde{w}_{1 \eta}^{v} U_{X}^{\eta}$ and $\tilde{w}_{3 \eta}^{v} U_{X}^{\eta}$ do not commute with each other.

4. The recursion operator and the higher Hamiltonian structures. We now use the symplectic operator (2.10) for nondegenerate bracket $\hat{J}_{(0)}$ and consider the recursion operator $\hat{R}_{\mu}^{v}=\hat{J}_{(1)}^{v \tau} \hat{\Omega}_{(0) \tau \mu}$ under the assumptions of Theorem 3.1.

We put $v^{\top}(U, \lambda)=n^{\top}(U, \lambda), \tau=1, \ldots, N, v^{N+k}(U, \lambda)=h_{(0)}^{k}(U, \lambda), k=1, \ldots, g_{0}$, $v_{0}^{s}(U) \equiv v^{s}(U, 0), E_{(0)}^{\tau}=\epsilon^{\tau}, \tau=1, \ldots, N$, and $E_{(0)}^{N+k}=e_{(0) k}, k=1, \ldots, g_{0}$ (where $n^{\tau}(U, \lambda)$ and $h_{(0)}^{k}(U, \lambda)$ are the functions from Theorem 3.1) and write the symplectic form $\hat{\Omega}_{(0) \tau \mu}$ as

$$
\hat{\Omega}_{(0) \tau \mu}=\sum_{k=1}^{N+g_{0}} E_{(0)}^{k} \frac{\partial v_{0}^{k}}{\partial U^{\tau}} D^{-1} \frac{\partial v_{0}^{k}}{\partial U^{\mu}}
$$

We can also write the operator $\hat{J}_{(0)}^{v \mu}$ as

$$
\hat{J}_{(0)}^{v \mu}=g_{(0)}^{v \mu} \frac{d}{d X}+b_{(0) \eta}^{v \mu} U_{X}^{\eta}+\sum_{k=1}^{N+g_{0}} E_{(0)}^{k}\left[\hat{J}_{(0)}^{v \tau} \frac{\partial v_{0}^{k}}{\partial U^{\tau}}\right] D^{-1}\left[\hat{J}_{(0)}^{\mu \sigma} \frac{\partial v_{0}^{k}}{\partial U^{\sigma}}\right] .
$$


For $\hat{R}_{\mu}^{v}$, we have the expression

$$
\begin{aligned}
\hat{R}_{\mu}^{v}=\hat{J}_{(1)}^{\nu \tau} \hat{\Omega}_{(0) \tau \mu}= & \sum_{k=1}^{N+g_{0}} E_{(0)}^{k} g_{(1)}^{\nu \tau}\left(\frac{\partial v_{0}^{k}}{\partial U^{\tau}}\right)_{X} D^{-1} \frac{\partial v_{0}^{k}}{\partial U^{\mu}} \\
& +\sum_{k=1}^{N+g_{0}} E_{(0)}^{k} g_{(1)}^{\nu \tau} \frac{\partial v_{0}^{k}}{\partial U^{\tau}} \frac{\partial v_{0}^{k}}{\partial U^{\mu}} \\
& +\sum_{k=1}^{N+g_{0}} E_{(0)}^{k} b_{(1) \eta}^{\nu \tau} U_{X}^{\eta} \frac{\partial v_{0}^{k}}{\partial U^{\tau}} D^{-1} \frac{\partial v_{0}^{k}}{\partial U^{\mu}} \\
& +\sum_{s=1}^{g_{1}} \sum_{k=1}^{N+g_{0}} e_{(1) s} E_{(0)}^{k} w_{(1) s \eta}^{v} U_{X}^{\eta} D^{-1} w_{(1) s \zeta}^{\tau} U_{X}^{\zeta} \frac{\partial v_{0}^{k}}{\partial U^{\tau}} D^{-1} \frac{\partial v_{0}^{k}}{\partial U^{\mu}},
\end{aligned}
$$

where

$$
w_{(1) s \zeta}^{\tau} U_{X}^{\zeta} \frac{\partial v_{0}^{k}}{\partial U^{\tau}} \equiv\left(Q_{s}^{k}\right)_{X}=\frac{d}{d X} Q_{s}^{k}-Q_{s}^{k} \frac{d}{d X}
$$

for some $Q_{s}^{k}(U)$ according to Theorem 3.1.

We normalize the functions $Q_{s}^{k}(U)$ such that $Q_{s}^{k}(z)=0$, and we have

$$
\cdots D^{-1} \frac{d}{d X} Q_{s}^{k} \cdots=\cdots Q_{s}^{k} \cdots
$$

on $L\left(\mu^{N}, z\right)$.

Also, $d / d X D^{-1} \equiv \hat{I}$ on $L\left(\mathcal{M}^{N}, z\right)$, and

$$
\begin{gathered}
\sum_{k=1}^{N+g_{0}} E_{(0)}^{k} g_{(1)}^{\nu \tau}\left(\frac{\partial v_{0}^{k}}{\partial U^{\tau}}\right)_{X} D^{-1} \frac{\partial v_{0}^{k}}{\partial U^{\mu}}+\sum_{k=1}^{N+g_{0}} E_{(0)}^{k} b_{(1) \eta}^{\nu \tau} U_{X}^{\eta} \frac{\partial v_{0}^{k}}{\partial U^{\tau}} D^{-1} \frac{\partial v_{0}^{k}}{\partial U^{\mu}} \\
+\sum_{s=1}^{g_{1}} \sum_{k=1}^{N+g_{0}} e_{(1) s} E_{(0)}^{k} w_{(1) s \eta}^{v} U_{X}^{\eta} Q_{s}^{k} D^{-1} \frac{\partial v_{0}^{k}}{\partial U^{\mu}} \\
=\sum_{k=1}^{N+g_{0}} E_{(0)}^{k}\left[\hat{J}_{(1)}^{v \tau} \frac{\partial v_{0}^{k}}{\partial U^{\tau}}\right] D^{-1} \frac{\partial v_{0}^{k}}{\partial U^{\mu}} .
\end{gathered}
$$

Also, using the relation

$$
\sum_{k=1}^{N+g_{0}} E_{(0)}^{k} \frac{\partial v_{0}^{k}}{\partial U^{\mu}} Q_{s}^{k}=\hat{\Omega}_{(0) \mu \tau} w_{(1) s \eta}^{\tau} U_{X}^{\eta}=\frac{\partial h_{(1)}^{s}(U, 0)}{\partial U^{\mu}}=\frac{\partial h_{(1) 0}^{s}(U)}{\partial U^{\mu}}
$$

on $L\left(\mathcal{M}^{N}, z\right)$ (since $\partial h_{(1) q}^{s} / \partial U^{\mu}(z)=0, q \geq 0$ ), we can write

$$
\begin{aligned}
\hat{R}_{\mu}^{v} & =g_{(1)}^{\nu \tau} g_{(0) \tau \mu}+\sum_{k=1}^{N+g_{0}} E_{(0)}^{k}\left[\hat{J}_{(1)}^{v \tau} \frac{\partial v_{0}^{k}}{\partial U^{\tau}}\right] D^{-1} \frac{\partial v_{0}^{k}}{\partial U^{\mu}}-\sum_{s=1}^{g_{1}} e_{(1) s} w_{(1) s \eta}^{v} U_{X}^{\eta} D^{-1} \frac{\partial h_{(1) 0}^{s}}{\partial U^{\mu}} \\
& =V_{\mu}^{v}-\sum_{k=1}^{N+g_{0}} E_{(0)}^{k}\left[\hat{J}_{(0)}^{\nu \tau} \frac{\partial v_{1}^{k}}{\partial U^{\tau}}\right] D^{-1} \frac{\partial v_{0}^{k}}{\partial U^{\mu}}-\sum_{s=1}^{g_{1}} e_{(1) s}\left[\hat{J}_{(0)}^{\nu \tau} \frac{\partial h_{(1) 0}^{s}}{\partial U^{\tau}}\right] D^{-1} \frac{\partial h_{(1) 0}^{s}}{\partial U^{\mu}}
\end{aligned}
$$


where

$$
v^{k}(U, \lambda)=\sum_{q=0}^{+\infty} v_{q}^{k}(U) \lambda^{q}, \quad h_{(1)}^{s}(U, \lambda)=\sum_{q=0}^{+\infty} h_{(1) q}^{s}(U) \lambda^{q}
$$

and $V_{\mu}^{\nu}(U)=g_{(1)}^{v \tau}(U) g_{(0) \tau \mu}(U)$.

We mention here that, according to this definition, $\hat{R}_{\mu}^{v}$ will act from the left on the vector-fields (on $\mathscr{V}_{0}(z)$ ) and from the right on the gradients of functionals on $L\left(\mu^{N}, z\right)$.

THEOREM 4.1. Consider the nondegenerate pencil (3.1) with $\operatorname{det} g_{(0)}^{v \mu} \neq 0$ such that the form $Q_{\lambda}^{k s}$ is also nondegenerate on $W$ for small enough $\lambda \neq 0$. Then,

(I) any power $\left[\hat{R}^{n}\right], n \geq 1$ of the recursion operator can be written in the form

$$
\begin{aligned}
{\left[\hat{R}^{n}\right]_{\mu}^{v}=} & {\left[\hat{V}^{n}\right]_{\mu}^{v}+(-1)^{n} \sum_{k=1}^{N+g_{0}} E_{(0)}^{k}\left(\sum_{s=1}^{n}\left[\hat{J}_{(0)}^{v \tau} \frac{\partial v_{s}^{k}}{\partial U^{\tau}}\right] D^{-1} \frac{\partial v_{n-s}^{k}}{\partial U^{\mu}}\right) } \\
& +(-1)^{n} \sum_{k=1}^{g_{1}} e_{(1) k}\left(\sum_{s=1}^{n}\left[\hat{J}_{(0)}^{v \tau} \frac{\partial h_{(1), s-1}^{k}}{\partial U^{\tau}}\right] D^{-1} \frac{\partial h_{(1), n-s}^{k}}{\partial U^{\mu}}\right)
\end{aligned}
$$

(II) the higher Hamiltonian structures $\hat{J}_{(n)}^{v \mu}=\left[\hat{R}^{n}\right]_{\xi}^{v} \hat{J}_{(0)}^{\xi \mu}$ can be written on $\mathscr{F}_{0}(z)$ in the following weakly nonlocal form:

$$
\begin{aligned}
\hat{J}_{(n)}^{v \mu}= & {\left[\hat{V}^{n}\right]_{\xi}^{v} g_{(0)}^{\xi \mu} \frac{d}{d X}+\left[\hat{V}^{n}\right]_{\xi}^{v} b_{(0) \eta}^{\xi \mu} U_{X}^{\eta} } \\
& +(-1)^{n} \sum_{k=1}^{N+g_{0}} E_{(0)}^{k}\left(\sum_{s=1}^{n}\left[\hat{J}_{(0)}^{v \tau} \frac{\partial v_{s}^{k}}{\partial U^{\tau}}\right] \frac{\partial v_{n-s}^{k}}{\partial U^{\xi}} g_{(0)}^{\xi \mu}\right) \\
& +(-1)^{n} \sum_{k=1}^{g_{1}} e_{(1) k}\left(\sum_{s=1}^{n}\left[\hat{J}_{(0)}^{v \tau} \frac{\partial h_{(1), s-1}^{k}}{\partial U^{\tau}}\right] \frac{\partial h_{(1), n-s}^{k}}{\partial U^{\xi}} g_{(0)}^{\xi \mu}\right) \\
& +(-1)^{n+1} \sum_{k=1}^{N+g_{0}} E_{(0)}^{k}\left(\sum_{s=1}^{n-1}\left[\hat{J}_{(0)}^{v \tau} \frac{\partial v_{s}^{k}}{\partial U^{\tau}}\right] D^{-1}\left[\hat{J}_{(0)}^{\mu \xi} \frac{\partial v_{n-s}^{k}}{\partial U^{\xi}}\right]\right) \\
& +(-1)^{n+1} \sum_{k=1}^{g_{1}} e_{(1) k}\left(\sum_{s=1}^{n}\left[\hat{J}_{(0)}^{v \tau} \frac{\partial h_{(1), s-1}^{k}}{\partial U^{\tau}}\right] D^{-1}\left[\hat{J}_{(0)}^{\mu \xi} \frac{\partial h_{(1), n-s}^{k}}{\partial U^{\xi}}\right]\right)
\end{aligned}
$$

for $n \geq 2$;

(III) all the "negative" symplectic forms

$$
\hat{\Omega}_{(-n) v \mu}=\hat{\Omega}_{(0) v \mu}\left[\hat{R}^{n}\right]_{\mu}^{\xi}, \quad n \geq 1
$$

can be represented on $\mathscr{V}_{0}(z)$ in the form

$$
\begin{aligned}
\hat{\Omega}_{(-n) v \mu}= & (-1)^{n} \sum_{k=1}^{N+g_{0}} E_{(0)}^{k}\left(\sum_{s=0}^{n} \frac{\partial v_{s}^{k}}{\partial U^{v}} D^{-1} \frac{\partial v_{n-s}^{k}}{\partial U^{\mu}}\right) \\
& +(-1)^{n} \sum_{k=1}^{g_{1}} e_{(1) k}\left(\sum_{s=1}^{n} \frac{\partial h_{(1), s-1}^{k}}{\partial U^{v}} D^{-1} \frac{\partial h_{(1), n-s}^{k}}{\partial U^{\mu}}\right) .
\end{aligned}
$$


Proof. (I) By induction, we have

$$
\begin{aligned}
\hat{R}_{\xi}^{v}\left[\hat{R}^{n}\right]_{\mu}^{\xi}= & {\left[\hat{V}^{n+1}\right]_{\mu}^{v}+(-1)^{n} \sum_{k=1}^{N+g_{0}} E_{(0)}^{k}\left(\sum_{s=1}^{n}\left[\hat{R}_{\xi}^{v} \hat{J}_{(0)}^{\xi \sigma} \frac{\partial v_{s}^{k}}{\partial U^{\sigma}}\right] D^{-1} \frac{\partial v_{n-s}^{k}}{\partial U^{\mu}}\right) } \\
& +(-1)^{n} \sum_{k=1}^{g_{1}} e_{(1) k}\left(\sum_{s=1}^{n}\left[\hat{R}_{\xi}^{v} \hat{J}_{(0)}^{\xi \sigma} \frac{\partial h_{(1), s-1}^{k}}{\partial U^{\sigma}}\right] D^{-1} \frac{\partial h_{(1), n-s}^{k}}{\partial U^{\mu}}\right) \\
& +(-1)^{n} \sum_{q=1}^{N+g_{0}} \sum_{k=1}^{N+g_{0}} E_{(0)}^{q}\left(\sum_{s=1}^{n}\left[\hat{J}_{(0)}^{v \tau} \frac{\partial v_{1}^{q}}{\partial U^{\tau}}\right] D^{-1} P_{0 s}^{q k} \frac{\partial v_{n-s}^{k}}{\partial U^{\mu}}\right) \\
& +(-1)^{n} \sum_{q=1}^{N+g_{0}} \sum_{k=1}^{g_{1}} E_{(0)}^{q}\left(\sum_{s=1}^{n}\left[\hat{J}_{(0)}^{v \tau} \frac{\partial v_{1}^{q}}{\partial U^{\tau}}\right] D^{-1} Q_{0 s}^{q k} \frac{\partial h_{(1), n-s}^{k}}{\partial U^{\mu}}\right) \\
& -\sum_{q=1}^{N+g_{0}} E_{(0)}^{q}\left[\hat{J}_{(0)}^{v \tau} \frac{\partial v_{1}^{q}}{\partial U^{\tau}}\right] D^{-1} \frac{\partial v_{0}^{q}}{\partial U^{\xi}}\left[\hat{V}^{n}\right]_{\mu}^{\xi} \\
& +(-1)^{n} \sum_{q=1}^{g_{1}} \sum_{k=1}^{N+g_{0}} e_{(1) q}\left(\sum_{s=1}^{n}\left[\hat{J}_{(0)}^{v \tau} \frac{\partial h_{(1), 0}^{q}}{\partial U^{\tau}}\right] D^{-1} S_{0 s}^{q k} \frac{\partial v_{n-s}^{k}}{\partial U^{\mu}}\right) \\
& +(-1)^{n} \sum_{q=1}^{g_{1}} \sum_{k=1}^{g_{1}} e_{(1) q}\left(\sum_{s=1}^{n}\left[\hat{J}_{(0)}^{v \tau} \frac{\partial h_{(1), 0}^{q}}{\partial U^{\tau}}\right] D^{-1} T_{0 s}^{q k} \frac{\partial h_{(1), n-s}^{k}}{\partial U^{\mu}}\right) \\
& \left.-\sum_{q=1}^{g_{1}} e_{(1) q}\left[\hat{J}_{(0)}^{v \tau} \frac{\partial h_{(1), 0}^{q}}{\partial U^{\tau}}\right] D^{-1} \frac{\partial h_{(1), 0}^{q}}{\partial U^{\xi}\left[\hat{V}^{n}\right.}\right]_{\mu}^{\xi},
\end{aligned}
$$

where we have

$$
\begin{gathered}
E_{(0)}^{k} \frac{\partial v_{0}^{q}}{\partial U^{\xi}}\left[\hat{J}_{(0)}^{\xi \sigma} \frac{\partial v_{s}^{k}}{\partial U^{\sigma}}\right] \equiv\left(P_{0 s}^{q k}\right)_{X}=\frac{d}{d X} P_{0 s}^{q k}-P_{0 s}^{q k} \frac{d}{d X}, \\
e_{(1) k} \frac{\partial v_{0}^{q}}{\partial U^{\xi}}\left[\hat{J}_{(0)}^{\xi \sigma} \frac{\partial h_{(1), s}^{k}}{\partial U^{\sigma}}\right] \equiv\left(Q_{0 s}^{q k}\right)_{X}=\frac{d}{d X} Q_{0 s}^{q k}-Q_{0 s}^{q k} \frac{d}{d X}, \\
E_{(0)}^{k} \frac{\partial h_{(1), 0}^{q}}{\partial U^{\xi}}\left[\hat{J}_{(0)}^{\xi \sigma} \frac{\partial v_{s}^{k}}{\partial U^{\sigma}}\right] \equiv\left(S_{0 s}^{q k}\right)_{X}=\frac{d}{d X} S_{0 s}^{q k}-S_{0 s}^{q k} \frac{d}{d X}, \\
e_{(1) k} \frac{\partial h_{(1), 0}^{q}}{\partial U^{\xi}}\left[\hat{J}_{(0)}^{\xi \sigma} \frac{\partial h_{(1), s}^{k}}{\partial U^{\sigma}}\right] \equiv\left(T_{0 s}^{q k}\right)_{X}=\frac{d}{d X} T_{0 s}^{q k}-T_{0 s}^{q k} \frac{d}{d X}
\end{gathered}
$$

for some functions $P_{0 s}^{q k}(U), Q_{0 s}^{q k}(U), S_{0 s}^{q k}(U), T_{0 s}^{q k}(U)$ according to Theorem 3.1, and we use the normalization

$$
P_{0 s}^{q k}(z)=Q_{0 s}^{q k}(z)=S_{0 s}^{q k}(z)=T_{0 s}^{q k}(z)=0 .
$$

From Theorem 3.1, we have

$$
\begin{aligned}
\frac{\partial v_{s}^{k}}{\partial U^{\tau}}(z) & =0, \quad s \geq 1 \quad\left(\text { actually } \frac{\partial h_{(0), 0}^{k}}{\partial U^{\tau}}(z)=0 \text { also }\right), \\
\frac{\partial h_{(1), s-1}^{k}}{\partial U^{\tau}}(z) & =0, \quad s \geq 1 ;
\end{aligned}
$$


so, for $s \geq 1$, we can write

$$
\left[\hat{\Omega}_{(0) v \xi} \hat{J}_{(0)}^{\xi \tau} \frac{\partial v_{s}^{k}}{\partial U^{\tau}}\right]=\frac{\partial v_{s}^{k}}{\partial U^{v}}, \quad\left[\hat{\Omega}_{(0) v \xi} \hat{J}_{(0)}^{\xi \tau} \frac{\partial h_{(1), s-1}^{k}}{\partial U^{\tau}}\right]=\frac{\partial h_{(1), s-1}^{k}}{\partial U^{v}}
$$

on $L\left(M^{N}, z\right)$ and

$$
\begin{gathered}
{\left[\hat{R}_{\xi}^{v} \hat{J}_{(0)}^{\xi \tau} \frac{\partial v_{s}^{k}}{\partial U^{\tau}}\right]=\left[\hat{J}_{(1)}^{v \tau} \frac{\partial v_{s}^{k}}{\partial U^{\tau}}\right]=-\left[\hat{J}_{(0)}^{v \tau} \frac{\partial v_{s+1}^{k}}{\partial U^{\tau}}\right],} \\
{\left[\hat{R}_{\xi}^{v} \hat{J}_{(0)}^{\xi \tau} \frac{\partial h_{(1), s-1}^{k}}{\partial U^{\tau}}\right]=\left[\hat{J}_{(1)}^{v \tau} \frac{\partial h_{(1), s-1}^{k}}{\partial U^{\tau}}\right]=-\left[\hat{J}_{(0)}^{v \tau} \frac{\partial h_{(1), s}^{k}}{\partial U^{\tau}}\right]}
\end{gathered}
$$

according to Theorem 2.2.

We now multiply the equalities

$$
\left[\hat{J}_{(1)}^{\xi \tau} \frac{\partial v_{s}^{k}}{\partial U^{\tau}}\right]=-\left[\hat{J}_{(0)}^{\xi \tau} \frac{\partial v_{s+1}^{k}}{\partial U^{\tau}}\right], \quad\left[\hat{J}_{(1)}^{\xi \tau} \frac{\partial h_{(1), s}^{k}}{\partial U^{\tau}}\right]=-\left[\hat{J}_{(0)}^{\xi \tau} \frac{\partial h_{(1), s+1}^{k}}{\partial U^{\tau}}\right]
$$

by $\hat{\Omega}_{(0) v \xi}$ from the left. Since

$$
\frac{\partial v_{s+1}^{k}}{\partial U^{\tau}}(z)=\frac{\partial h_{(1), s+1}^{k}}{\partial U^{\tau}}(z)=0, \quad s \geq 0
$$

we get again from Theorem 2.2

$$
\begin{gathered}
\frac{\partial v_{s+1}^{k}}{\partial U^{v}}=-\left[\hat{\Omega}_{(0) v \xi} \hat{J}_{(1)}^{\xi \tau} \frac{\partial v_{s}^{k}}{\partial U^{\tau}}\right]=-\left[\frac{\partial v_{s}^{k}}{\partial U^{\tau}} \hat{R}_{v}^{\tau}\right], \quad s \geq 0, \\
\frac{\partial h_{(1), s+1}^{k}}{\partial U^{v}}=-\left[\hat{\Omega}_{(0) v \xi} \hat{J}_{(1)}^{\xi \tau} \frac{\partial h_{(1), s}^{k}}{\partial U^{\tau}}\right]=-\left[\frac{\partial h_{(1), s}^{k}}{\partial U^{\tau}} \hat{R}_{v}^{\tau}\right], \quad s \geq 0
\end{gathered}
$$

(action from the right).

Using the relation

$$
\left[f_{X} D^{-1}\right]=-f(X)
$$

for the action from the right of $D^{-1}$ on any $f(U)$ such that $f(z)=0$, we get that the last six terms in the expression for $\hat{R}_{\xi}^{v}\left[\hat{R}^{n}\right]_{\mu}^{\xi}$ can be written as

$$
\begin{aligned}
& -\sum_{q=1}^{N+g_{0}} E_{(0)}^{q}\left[\hat{J}_{(0)}^{v \tau} \frac{\partial v_{1}^{q}}{\partial U^{\tau}}\right] D^{-1}\left[\frac{\partial v_{0}^{q}}{\partial U^{\xi}}\left(\hat{R}^{n}\right)_{\mu}^{\xi}\right]-\sum_{q=1}^{g_{1}} e_{(1) q}\left[\hat{J}_{(0)}^{v \tau} \frac{\partial h_{(1), 0}^{q}}{\partial U^{\tau}}\right] D^{-1}\left[\frac{\partial h_{(1), 0}^{q}}{\partial U^{\xi}}\left(\hat{R}^{n}\right)_{\mu}^{\xi}\right] \\
& =(-1)^{n+1} \sum_{q=1}^{N+g_{0}} E_{(0)}^{q}\left[\hat{J}_{(0)}^{v \tau} \frac{\partial v_{1}^{q}}{\partial U^{\tau}}\right] D^{-1} \frac{\partial v_{n}^{q}}{\partial U^{\mu}}+(-1)^{n+1} \sum_{q=1}^{g_{1}} e_{(1) q}\left[\hat{J}_{(0)}^{v \tau} \frac{\partial h_{(1), 0}^{q}}{\partial U^{\tau}}\right] D^{-1} \frac{\partial h_{(1), n}^{q}}{\partial U^{\mu}} .
\end{aligned}
$$

Now, using relations (4.19) and (4.20), we get part (I) of the theorem. 
(II) To avoid much calculations, we just write that according to Theorem 3.1 we have the relations

$$
\begin{gathered}
E_{(0)}^{k} \frac{\partial v_{n-s}^{q}}{\partial U^{\xi}}\left[\hat{J}_{(0)}^{\xi \sigma} \frac{\partial v_{0}^{k}}{\partial U^{\sigma}}\right] \equiv\left(P_{n-s, 0}^{q k}\right)_{X}=\frac{d}{d X} P_{n-s, 0}^{q k}-P_{n-s, 0}^{q k} \frac{d}{d X}, \\
E_{(0)}^{k} \frac{\partial h_{(1), n-s}^{q}}{\partial U^{\xi}}\left[\hat{J}_{(0)}^{\xi \sigma} \frac{\partial v_{0}^{k}}{\partial U^{\sigma}}\right] \equiv\left(S_{n-s, 0}^{q k}\right)_{X}=\frac{d}{d X} S_{n-s, 0}^{q k}-S_{n-s, 0}^{q k} \frac{d}{d X}
\end{gathered}
$$

for some $P_{n-s, 0}^{q k}(U), S_{n-s, 0}^{q k}(U), P_{n-s, 0}^{q k}(z)=0$, and $S_{n-s, 0}^{q k}(z)=0$, and so, the expression $\hat{J}_{(n)}=\hat{R}^{n} \hat{J}_{(0)}$ can be written according on $\mathscr{F}_{0}(z)$ to (4.10) and (4.2) and Theorem 2.2 as

$$
\begin{aligned}
\hat{J}_{(n)}^{v \mu}= & \left(\text { local part of } \hat{R}^{n}\right) \times\left(\text { local part of } \hat{J}_{(0)}\right) \\
& +(-1)^{n} \sum_{k=1}^{N+g_{0}} E_{(0)}^{k}\left(\sum_{s=1}^{n}\left[\hat{J}_{(0)}^{v \tau} \frac{\partial v_{s}^{k}}{\partial U^{\tau}}\right] \frac{\partial v_{n-s}^{k}}{\partial U^{\xi}} g_{(0)}^{\xi \mu}\right) \\
& +(-1)^{n} \sum_{k=1}^{g_{1}} e_{(1) k}\left(\sum_{s=1}^{n}\left[\hat{J}_{(0)}^{v \tau} \frac{\partial h_{(1), s-1}^{k}}{\partial U^{\tau}}\right] \frac{\partial h_{(1), n-s}^{k}}{\partial U^{\xi}} g_{(0)}^{\xi \mu}\right) \\
& +\sum_{q=1}^{N+g_{0}} E_{(0)}^{q}\left[\left(\hat{R}^{n}\right)_{\xi}^{v} \hat{J}_{(0)}^{\xi \tau} \frac{\partial v_{0}^{q}}{\partial U^{\tau}}\right] D^{-1}\left[\hat{J}_{(0)}^{\mu \sigma} \frac{\partial v_{0}^{q}}{\partial U^{\sigma}}\right] \\
& -(-1)^{n} \sum_{k=1}^{N+g_{0}} E_{(0)}^{k}\left(\sum_{s=1}^{n}\left[\hat{J}_{(0)}^{v \tau} \frac{\partial v_{s}^{k}}{\partial U^{\tau}}\right] D^{-1}\left[\hat{J}_{(0)}^{\mu \sigma} \frac{\partial v_{n-s}^{k}}{\partial U^{\sigma}}\right]\right) \\
& -(-1)^{n} \sum_{k=1}^{g_{1}} e_{(1) k}\left(\sum_{s=1}^{n}\left[\hat{J}_{(0)}^{v \tau} \frac{\partial h_{(1), s-1}^{k}}{\partial U^{\tau}}\right] D^{-1}\left[\hat{J}_{(0)}^{\mu \sigma} \frac{\partial h_{(1), n-s}^{k}}{\partial U^{\sigma}}\right]\right)
\end{aligned}
$$

(since $\hat{J}_{(0)}$ is skew-symmetric, its action from the right differs by sign from the action from the left). Now, using (4.19), we get part (II) of the theorem. We also mention that it is important that we consider the space $\mathscr{F}_{0}(z)$ to use the equality

$$
D^{-1} \frac{d}{d X} \frac{\partial v_{0}^{k}}{\partial U^{\xi}}=\frac{\partial v_{0}^{k}}{\partial U^{\xi}}
$$

for $k=1, \ldots, N$.

(III) We have

$$
\hat{\Omega}_{(-n) v \mu}=\hat{\Omega}_{(0) v \xi}\left(\hat{R}^{n}\right)_{\mu}^{\xi} .
$$

Again, using the functions $P_{0 s}^{q k}(U)$ and $Q_{0 s}^{q k}(U)$, we can write

$$
\begin{aligned}
\hat{\Omega}_{(-n) v \mu}= & \sum_{k=1}^{N+g_{0}} E_{(0)}^{k} \frac{\partial v_{0}^{k}}{\partial U^{v}} D^{-1}\left[\frac{\partial v_{0}^{k}}{\partial U^{\xi}}\left(\hat{R}^{n}\right)_{\mu}^{\xi}\right] \\
& +(-1)^{n} \sum_{k=1}^{N+g_{0}} E_{(0)}^{k}\left(\sum_{s=1}^{n}\left[\hat{\Omega}_{(0) v \xi} \hat{J}_{(0)}^{\xi \tau} \frac{\partial v_{s}^{k}}{\partial U^{\tau}}\right] D^{-1} \frac{\partial v_{n-s}^{k}}{\partial U^{\mu}}\right) \\
& +(-1)^{n} \sum_{k=1}^{g_{1}} e_{(1) k}\left(\sum_{s=1}^{n}\left[\hat{\Omega}_{(0) v \xi} \hat{J}_{(0)}^{\xi \tau} \frac{\partial h_{(1), s-1}^{k}}{\partial U^{\tau}}\right] D^{-1} \frac{\partial h_{(1), n-s}^{k}}{\partial U^{\mu}}\right) .
\end{aligned}
$$


Since

$$
\frac{\partial v_{s}^{k}}{\partial U^{\tau}}(z)=0, \quad \frac{\partial h_{(1), s-1}^{k}}{\partial U^{\tau}}=0, \quad s \geq 1
$$

we get part (III) using Theorem 2.2 and (4.19) on $L\left(M^{N}, z\right)$.

We also mention that if both $\operatorname{det} g_{(0)}^{\nu \mu} \neq 0$ and $\operatorname{det} g_{(1)}^{\nu \mu} \neq 0$ (and the form $Q$ in nondegenerate on $\mathcal{W})$, then also the series of "negative" Hamiltonian operators $\hat{J}_{(-n)}=$ $\hat{R}^{-n} \hat{J}_{(0)}$ and "positive" symplectic forms $\hat{\Omega}_{(n)}=\hat{\Omega}_{(0)} \hat{R}^{-n}$ will be weakly nonlocal. This situation takes place, for example, in the Hamiltonian structures of Whitham systems for KdV, NLS, and SG hierarchies. The local bi-Hamiltonian structure for the averaged KdV hierarchy was constructed in [6] (see also [7, 8]) using the (Dubrovin-Novikov) procedure of averaging of local field-theoretical brackets for Gardner-Zakharov-Faddeev and Magri brackets. Both metrics of the corresponding DN-brackets are nondegenerate in this case (and there is no requirements on $Q$ ). Also, in [7, 8], the local bracket for the averaged "V-Gordon" equation

$$
\varphi_{t t}-\varphi_{x x}+V^{\prime}(\varphi)=0
$$

which is the generalization of SG system using the same procedure was constructed. In [32], all the brackets for averaged KdV, NLS, and SG hierarchies, having local (DN) or constant curvature (MF) form, were enumerated using a nice differential-geometrical approach. It can be shown that all the pencils represented in $[6,7,8,32]$ actually satisfy the requirements of Theorems 3.1 and 4.1. The recursion operator approach for the local bi-Hamiltonian structure for the averaged KdV hierarchy (in the diagonal form) was investigated in [1, 2], and all the "positive" operators $\hat{J}_{(n)}$ were explicitly found in [1] in this case. In [23, 24], the general procedure of averaging of brackets (1.23), which gives the weakly nonlocal Hamiltonian operators for the averaged systems with Hamiltonian structure (1.23), was constructed. For many "integrable" systems, this method also gives all the "positive" weakly nonlocal Poisson brackets of Ferapontov type for the corresponding Whitham hierarchy. However, we see here that the "negative" Hamiltonian operators and Symplectic structures for the averaged KdV, NLS, and SG also should be weakly nonlocal according to Theorems 3.1 and 4.1. We believe that there should be a good procedure of averaging of "negative" Hamiltonian operators for the integrable systems giving the brackets of Ferapontov type and the general averaging procedure for the weakly nonlocal symplectic structures

$$
\hat{\Omega}_{\nu \mu}(x, y)=\sum_{k=1}^{N} C_{\nu \mu}^{(k)}\left(\varphi, \varphi_{x}, \ldots\right) \delta^{(k)}(x-y)+\sum_{k, s=1}^{G} d_{k s} \frac{\delta H_{(k)}}{\delta \varphi(x)} v(x-y) \frac{\delta H_{(s)}}{\delta \varphi(y)}
$$

giving the weakly nonlocal symplectic structures and the corresponding F-brackets for the Whitham systems.

We also believe that the general compatible F-brackets should be important for the integration of nondiagonalizable (bi-Hamiltonian) systems which cannot be integrated by Tsarëv's method. 
We also mention that the requirement of nondegeneracy of form $Q$ on $\mathcal{W}$ is also important in Theorem 4.1. As such, it is possible to show that the "negative" and "positive" Poisson structures corresponding to the pair of operators (3.36) will not be weakly nonlocal.

\section{REFERENCES}

[1] V. L. Alekseev, On non-local Hamiltonian operators of hydrodynamic type connected with Whitham's equations, Russian Math. Surveys 50 (1995), no. 6, 1253-1255.

[2] V. L. Alekseev and M. V. Pavlov, Hamiltonian structures of the Whitham equations, preprint, 1994.

[3] L. V. Bogdanov and E. V. Ferapontov, A nonlocal Hamiltonian formalism for semiHamiltonian systems of hydrodynamic type, Theoret. and Math. Phys. 116 (1998), no. 1, 829-835.

[4] B. A. Dubrovin, Integrable systems in topological field theory, Nuclear Phys. B 379 (1992), no. 3, 627-689.

[5] __ Flat pencils of metrics and Frobenius manifolds, Integrable Systems and Algebraic Geometry (Kobe/Kyoto, 1997) (M.-H. Saito et al., eds.), World Scientific Publishing, New Jersey, 1998, pp. 47-72.

[6] B. A. Dubrovin and S. P. Novikov, Hamiltonian formalism of one-dimensional systems of the hydrodynamic type and the Bogolyubov-Whitham averaging method, Soviet Math. Dokl. 27 (1983), no. 3, 665-669.

[7] _ Hydrodynamics of weakly deformed soliton lattices. Differential geometry and Hamiltonian theory, Russian Math. Surveys 44 (1989), no. 6, 35-124.

[8] _ Hydrodynamics of Soliton Lattices, Soviet Scientific Reviews, Section C: Mathematical Physics Reviews, vol. 9, Harwood Academic Publishers GmbH, Yverdon, 1993.

[9] B. A. Dubrovin and Y. Zhang, Normal forms of hierarchies of integrable PDEs, Frobenius manifolds and Gromov-Witten invariants, preprint 2001 http://arxiv.org/abs/math.DG/0108160.

[10] B. Enriquez, A. Orlov, and V. Rubtsov, Higher Hamiltonian structures (the $\mathrm{sl}_{2}$ case), JETP Lett. 58 (1993), no. 8, 658-664.

[11] E. V. Ferapontov, Differential geometry of nonlocal Hamiltonian operators of hydrodynamic type, Funct. Anal. Appl. 25 (1991), no. 3, 195-204.

[12] _ Dirac reduction of the Hamiltonian operator $\delta^{I J}(d / d x)$ to a submanifold of Euclidean space with flat normal connection, Funct. Anal. Appl. 26 (1992), no. 4, 298300.

[13] _ Nonlocal matrix Hamiltonian operators. Differential geometry and applications, Theoret. and Math. Phys. 91 (1992), no. 3, 642-649.

[14]__ On integrability of $3 \times 3$ semi-Hamiltonian hydrodynamic type systems $u_{t}^{i}=$ $v_{j}^{i}(u) u_{x}^{j}$ which do not possess Riemann invariants, Phys. D 63 (1993), no. 1-2, $50-70$.

[15]___ On the matrix Hopf equation and integrable Hamiltonian systems of hydrodynamic type, which do not possess Riemann invariants, Phys. Lett. A 179 (1993), no. 6, 391397.

[16] _ Nonlocal Hamiltonian operators of hydrodynamic type: differential geometry and applications, Topics in Topology and Mathematical Physics, Amer. Math. Soc. Transl. Ser. 2, vol. 170, American Mathematical Society, Rhode Island, 1995, pp. 3358.

[17] _ Compatible Poisson brackets of hydrodynamic type, J. Phys. A 34 (2001), no. 11, 2377-2388.

[18] N. I. Grinberg, On Poisson brackets of hydrodynamic type with degenerate metric, Uspekhi Mat. Nauk 40 (1985), no. 4(244), 217-218 (Russian). 
[19] I. M. Krichever, Algebraic-geometric n-orthogonal curvilinear coordinate systems and the solution of associativity equations, Funct. Anal. Appl. 31 (1997), no. 1, 25-39.

[20] F. Magri, A simple model of the integrable Hamiltonian equation, J. Math. Phys. 19 (1978), no. $5,1156-1162$.

[21] A. Ya. Maltsev, The averaging of local field-theoretic Poisson brackets, Russian Math. Surveys 52 (1997), no. 2, 409-411.

[22] Conservation of Hamiltonian structures in Whitham's averaging method, Izv. Math. 63 (1999), no. 6, 1171-1201.

[23] _ Non-local Poisson brackets and Whitham's method, Russian Math. Surveys 54 (1999), no. 6, 1252-1253.

[24] _ The averaging of nonlocal Hamiltonian structures in Whitham's method, Int. J. Math. Math. Sci. 30 (2002), no. 7, 399-434.

[25] A. Ya. Maltsev and S. P. Novikov, On the local systems Hamiltonian in the weakly non-local Poisson brackets, Phys. D 156 (2001), no. 1-2, 53-80.

[26] A. Ya. Maltsev and M. V. Pavlov, On Whitham's averaging method, Funct. Anal. Appl. 29 (1995), no. 1, 6-19.

[27] O. I. Mokhov, Compatible and almost compatible pseudo-Riemannian metrics, Funktsional. Anal. i Prilozhen. 35 (2001), no. 2, 24-36, 95 (Russian).

[28] On the integrability of equations for nonsingular pairs of compatible flat metrics, Teoret. Mat. Fiz. 130 (2002), no. 2, 233-250 (Russian).

[29] O. I. Mokhov and E. V. Ferapontov, Non-local Hamiltonian operators of hydrodynamic type related to metrics of constant curvature, Russian Math. Surveys 45 (1990), no. 3, 218-219.

[30] S. P. Novikov and A. Ya. Maltsev, Liouville form of averaged Poisson brackets, Russian Math. Surveys 48 (1993), no. 1, 155-157.

[31] M. V. Pavlov, Elliptic coordinates and multi-Hamiltonian structures of systems of hydrodynamic type, Russian Acad. Sci. Dokl. Math. 50 (1995), no. 3, 374-377.

[32] _ Multi-Hamiltonian structures of the Whitham equations, Russian Acad. Sci. Dokl. Math. 50 (1995), no. 2, 220-223.

[33] V. V. Sokolov, On the Hamiltonian property of the Krichever-Novikov equation, Soviet Math. Dokl. 30 (1984), no. 1, 44-46.

[34] S. P. Tsarëv, On Poisson brackets and one-dimensional Hamiltonian systems of hydrodynamic type, Soviet Math. Dokl. 31 (1985), no. 3, 488-491.

[35] _ The geometry of Hamiltonian systems of hydrodynamic type. The generalized hodograph method, Math. USSR-Izv. 37 (1991), no. 2, 397-419.

[36] V. E. Zakharov, Description of the n-orthogonal curvilinear coordinate systems and Hamiltonian integrable systems of hydrodynamic type. I. Integration of the Lamé equations, Duke Math. J. 94 (1998), no. 1, 103-139.

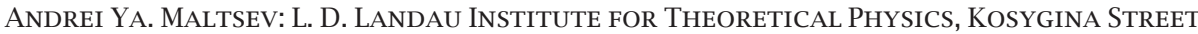

2, Moscow 117940, Russia

Current address: UnIVERSITY OF MARYLAND, COLlEgE PARK, MD 20742, USA

E-mail address: ma1tsev@itp.ac.ru, ma1tsev@math.umd.edu 


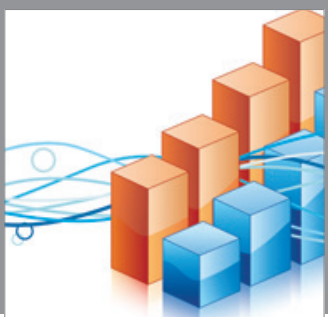

Advances in

Operations Research

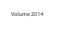

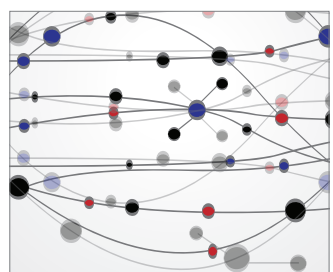

\section{The Scientific} World Journal
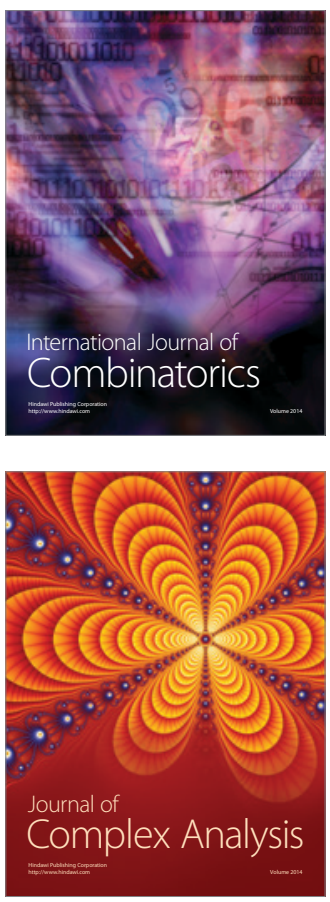

International Journal of

Mathematics and

Mathematical

Sciences
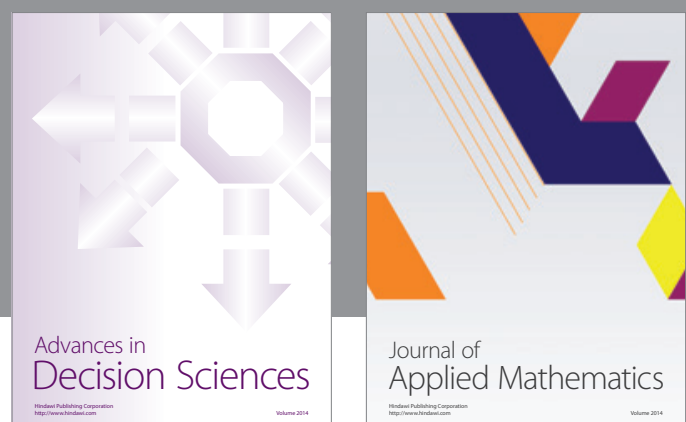

Journal of

Applied Mathematics
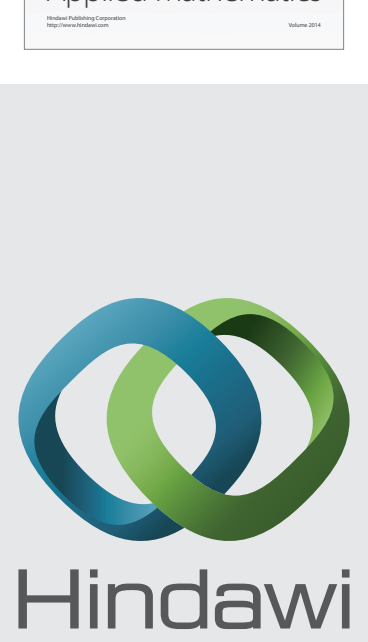

Submit your manuscripts at http://www.hindawi.com
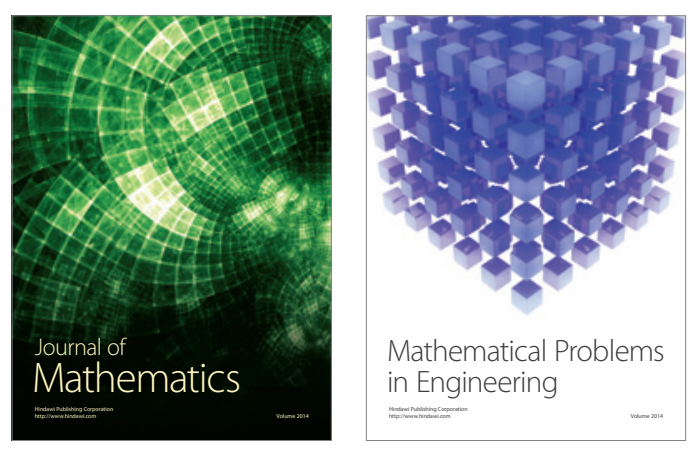

Mathematical Problems in Engineering
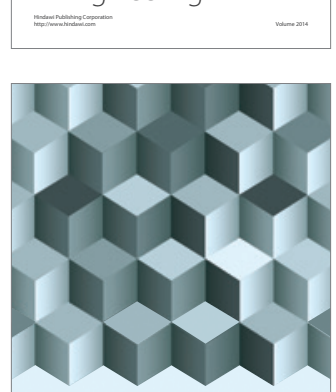

Journal of

Function Spaces
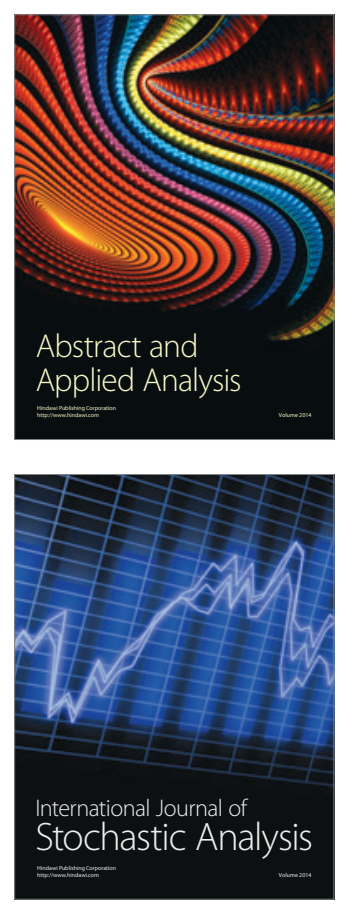

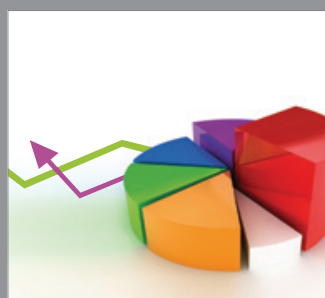

ournal of

Probability and Statistics

Promensencen
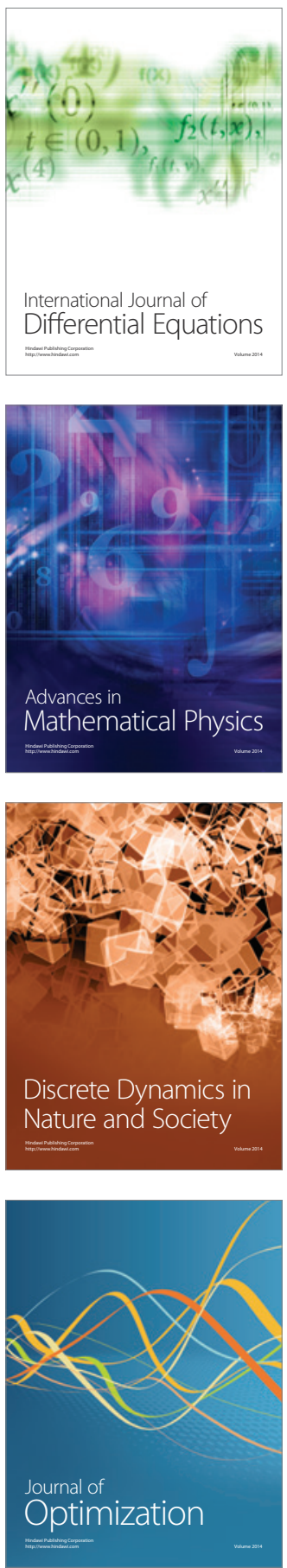